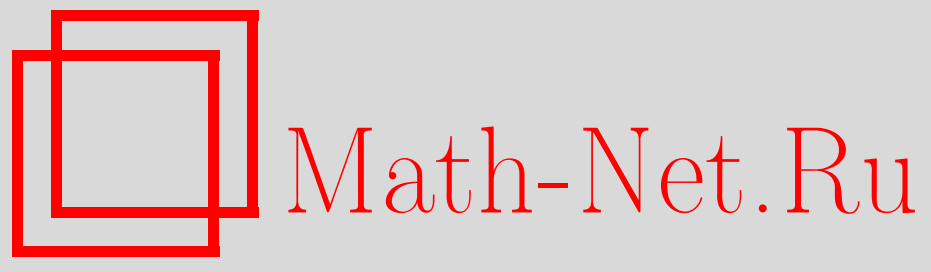

А. А. Васильева, Поперечники весовых классов Соболева на области с пиком, Матем. сб., 2015, том 206, номер 10, 37-70

DOI: https://doi.org/10.4213/sm8461

Использование Общероссийского математического портала Math-Net.Ru подразумевает, что вы прочитали и согласны с пользовательским соглашением http://www.mathnet.ru/rus/agreement

Параметры загрузки:

IP : 54.198 .187 .58

26 апреля 2023 г., 17:49:00

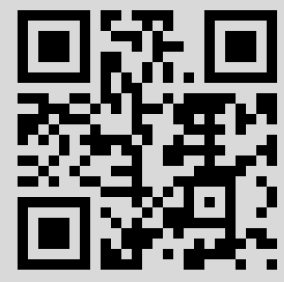




\title{
А. А. Васильева \\ Поперечники весовых классов Соболева на области с пиком
}

\begin{abstract}
Получены порядковые оценки колмогоровских, гельфандовских и линейных поперечников единичных шаров весовых пространств Соболева на области с пиком в весовом пространстве Лебега. Рассматриваются некоторые предельные условия на параметры, при которых оценки поперечников могут отличаться от оценок в случае единичных весов и области с липшицевой границей.
\end{abstract}

Библиография: 52 названия.

Ключевые слова: поперечники, области с пиком, весовые пространства Соболева.

DOI: $10.4213 / \mathrm{sm} 8461$

\section{§ 1. Введение}

Известно (см., например, [1]-[6]), что для областей $\Omega$, имеющих нулевые углы, условия компактности вложения пространства Соболева $\mathscr{W}_{p}^{r}(\Omega)$ в пространство $L_{q}(\Omega)$ отличаются от условий компактности вложения в случае области с липшицевой границей. В связи с этим возникает задача: как особенности границы области влияют на такие характеристики компактного вложения, как поперечники? В работе О. В. Бесова [7] показано, что если область имеет вид

$$
\Omega=\left\{\left(x^{\prime}, x_{d}\right): 0<x_{d}<\frac{1}{2}, \frac{x^{\prime}}{\varphi\left(x_{d}\right)} \in B^{d-1}\right\}
$$

где $B^{d-1} \subset \mathbb{R}^{d-1}$ - шар радиуса единица с центром в нуле,

$$
\varphi(t)=t^{\sigma}, \quad 1<p<q<\infty, \quad \sigma>1, \quad r+(\sigma(d-1)+1)\left(\frac{1}{q}-\frac{1}{p}\right)>0,
$$

то порядки колмогоровских поперечников единичного шара пространства $\mathscr{W}_{p}^{r}(\Omega)$ в пространстве $L_{q}(\Omega)$ такие же, как для областей с липшицевой границей. Если

$$
\varphi(t)=t^{\sigma}, \quad r+(\sigma(d-1)+1)\left(\frac{1}{q}-\frac{1}{p}\right)=0,
$$

то при $1<p<q<\infty, \sigma>1$ компактного вложения нет. Если

$$
\varphi(t)=t^{\sigma}|\log t|^{\theta}, \quad r+(\sigma(d-1)+1)\left(\frac{1}{q}-\frac{1}{p}\right)=0, \quad \theta>0,
$$

Исследование выполнено за счет гранта Российского научного фонда (проект № 14-50-00005).

(C) А. А. ВАСиЛьевА, 2015 
то из результатов В. Г. Мазьи и С. В. Поборчего (см. [6]) следует, что вложение компактное. Здесь будут получены порядковые оценки поперечников в этом предельном случае. При малых $\theta$ оценки будут отличаться от оценок для областей с липшицевой границей. Кроме того, будет получено обобщение этого результата на случай весовых пространств.

Дадим определение весового пространства Соболева и весового пространства Лебега.

Пусть $d \in \mathbb{N}, \Omega \subset \mathbb{R}^{d}$ - ограниченная область (открытое связное множество), $g, v: \Omega \rightarrow(0, \infty)$ - измеримые функции. Для каждой измеримой векторнозначной функции $\psi: \Omega \rightarrow \mathbb{R}^{m}, \psi=\left(\psi_{k}\right)_{1 \leqslant k \leqslant m}, p \in[1, \infty]$, положим

$$
\|\psi\|_{L_{p}(\Omega)}=\left\|\max _{1 \leqslant k \leqslant m}\left|\psi_{k}\right|\right\|_{p}=\left(\int_{\Omega} \max _{1 \leqslant k \leqslant m}\left|\psi_{k}(x)\right|^{p} d x\right)^{\frac{1}{p}}
$$

(с соответствующим изменением при $p=\infty)$. Пусть $\bar{\beta}=\left(\beta_{1}, \ldots, \beta_{d}\right) \in \mathbb{Z}_{+}^{d}:=$ $(\mathbb{N} \cup\{0\})^{d},|\bar{\beta}|=\beta_{1}+\cdots+\beta_{d}$. Для каждой обобщенной функции $f$ на $\Omega$ обозначим $\nabla^{r} f=\left(\frac{\partial^{r} f}{\partial x^{\bar{\beta}}}\right)_{|\bar{\beta}|=r}$ (частные производные берутся в обобщенном смысле) и обозначим через $m_{r, d}$ число компонент векторнозначной обобщенной функции $\nabla^{r} f$. Положим

$$
W_{p, g}^{r}(\Omega)=\left\{f: \Omega \rightarrow \mathbb{R} \mid \exists \psi: \Omega \rightarrow \mathbb{R}^{m_{r, d}}:\|\psi\|_{L_{p}(\Omega)} \leqslant 1, \nabla^{r} f=g \cdot \psi\right\}
$$

(соответствующую функцию $\psi$ обозначим через $\frac{\nabla^{r} f}{g}$ ),

$$
\begin{gathered}
\mathscr{W}_{p, g}^{r}(\Omega)=\operatorname{span} W_{p, g}^{r}(\Omega), \\
\|f\|_{L_{q, v}(\Omega)}=\|f\|_{q, v}=\|f v\|_{L_{q}(\Omega)}, \quad L_{q, v}(\Omega)=\left\{f: \Omega \rightarrow \mathbb{R} \mid\|f\|_{q, v}<\infty\right\} .
\end{gathered}
$$

Назовем $\mathscr{W}_{p, g}^{r}(\Omega)$ весовым пространством Соболева. Тогда $\mathscr{W}_{p, 1}^{r}(\Omega)=\mathscr{W}_{p}^{r}(\Omega)-$ невесовое пространство Соболева; единичный шар в нем будем обозначать через $W_{p}^{r}(\Omega)$. Отметим, что если $g \in L_{p^{\prime}}^{\text {loc }}(\Omega)$, то $\nabla^{r} f \in L_{1}^{\text {loc }}\left(\Omega, \mathbb{R}^{m_{r, d}}\right)$; здесь и далее $p^{\prime}=\frac{p}{p-1}-$ сопряженный показатель.

Отметим, что множество $W_{p, g}^{r}(\Omega)$ содержит пространство полиномов степени не выше $r-1$ (обозначим его через $\mathscr{P}_{r-1}(\Omega)$ ). Поэтому задачу о непрерывном (компактном) вложении весового пространство Соболева в $L_{q, v}(\Omega)$ естественно поставить следующим образом: существует ли ограниченное (предкомпактное) в $L_{q, v}(\Omega)$ множество $M$ такое, что $W_{p, g}^{r}(\Omega) \subset \mathscr{P}_{r-1}(\Omega)+M$ ?

Свойства весовых пространств Соболева и их обобщений описаны в книгах [8]-[12] и в обзорной статье [13]. В. Г. Мазья (см. [14]) получил необходимое и достаточное условие вложения $\mathscr{W}_{p}^{1}(\Omega)$ в $L_{q}(\Omega)$ в терминах изопериметрических и емкостных неравенств. Ю.Г. Решетняк в [15], [16], О.В. Бесов в [1] и Б. Боярский в [17] показали, что для области $\Omega$ с условием Джона или с условием гибкого конуса условие непрерывного вложения $\mathscr{W}_{p}^{r}(\Omega)$ в $L_{q}(\Omega)$ такое же, как для области с липшицевой границей (условие Джона или условие гибкого конуса означает, что область не имеет нулевых внутренних углов; см. определение 1 ниже); в работе [18] этот результат распространен на области с более общим условием распадающегося гибкого конуса. Также интенсивно изучалась задача о вложении весовых и невесовых пространств Соболева на области с нерегулярной границей, имеющей нулевые углы (см., например, [1]-[6], [19]-[25]). 
Пусть $\varphi:(0,1) \rightarrow(0, \infty)$ - неубывающая липшицева функция такая, что $\lim _{z \rightarrow+0} \varphi(z)=\lim _{z \rightarrow+0} \varphi^{\prime}(z)=0, G \subset \mathbb{R}^{d-1}$ - ограниченная область. Обозначим

$$
D_{\varphi, G}=\left\{x=(y, z) \in \mathbb{R}^{d}: z \in\left(0, \frac{1}{2}\right), \frac{y}{\varphi(z)} \in G\right\} .
$$

В [6] был получен критерий непрерывного вложения $\mathscr{W}_{p}^{r}\left(D_{\varphi, G}\right)$ в $L_{q}\left(D_{\varphi, G}\right)$, где $G \subset \mathbb{R}^{d-1}$ - ограниченная область, удовлетворяющая условию конуса. В данной статье мы обобщаем этот критерий на случай весовых пространств Соболева с весами, зависящими только от последней переменной (см. теорему 2).

Дадим определение колмогоровского, линейного и гельфандовского поперечников.

Пусть $\left(X,\|\cdot\|_{X}\right)$ - нормированное пространство, $X^{*}$ - его сопряженное, $\mathscr{L}_{n}(X), n \in \mathbb{Z}_{+},-$семейство подпространств в $X$ размерности не выше $n$. Обозначим через $L(X, Y)$ пространство линейных непрерывных операторов из $X$ в нормированное пространство $Y$. Через $\mathrm{rk} A$ обозначим размерность образа оператора $A \in L(X, Y)$, а через $\|A\|_{X \rightarrow Y}-$ его норму.

Колмогоровским $n$-поперечником центрально-симметричного множества $M \subset X$ в пространстве $X$ называется величина

$$
d_{n}(M, X)=\inf _{L \in \mathscr{L}_{n}(X)} \sup _{x \in M} \inf _{y \in L}\|x-y\|_{X},
$$

линейным $n$-поперечником -

$$
\lambda_{n}(M, X)=\inf _{A \in L(X, X), \mathrm{rk}} \sup _{A \leqslant n}\|x-A x\|_{X},
$$

гельфандовским $n$-поперечником -

$$
\begin{aligned}
d^{n}(M, X) & =\inf _{x_{1}^{*}, \ldots, x_{n}^{*} \in X^{*}} \sup \left\{\|x\|: x \in M, x_{j}^{*}(x)=0,1 \leqslant j \leqslant n\right\} \\
& =\inf _{A \in L\left(X, \mathbb{R}^{n}\right)} \sup \{\|x\|: x \in M \cap \operatorname{ker} A\} .
\end{aligned}
$$

В работе [26] было дано определение строгих $s$-чисел линейного непрерывного оператора. В частности, числа Колмогорова оператора $A: X \rightarrow Y$ совпадают с колмогоровскими поперечниками $d_{n}\left(A\left(B_{X}\right), Y\right)$ (где $B_{X}$ - единичный шар пространства $X)$; если оператор компактен, то его аппроксимативные числа совпадают с линейными поперечниками $\lambda_{n}\left(A\left(B_{X}\right), Y\right)$, а числа Гельфанда с гельфандовскими поперечниками $d^{n}\left(A\left(B_{X}\right), Y\right)$ (см. работу С. Хейнриха [27]). Если $X$ и $Y$ равномерно выпуклые и равномерно гладкие и $A: X \rightarrow Y$ - ограниченный линейный оператор с тривиальным ядром и плотным в $Y$ образом, то числа Гельфанда оператора $A$ также равны $d^{n}\left(A\left(B_{X}\right), Y\right)$ (см. работу Д. Эдмундса и Я. Ланга [28]).

В 1960-1980-е гг. интенсивно изучались задачи об оценке поперечников функциональных классов в $L_{q}$ и конечномерных шаров $B_{p}^{m}$ в $l_{q}^{m}$ (см., например, книги [29]-[31], а также статьи [32]-[36]). Здесь $l_{q}^{m}, 1 \leqslant q \leqslant \infty,-$ пространство $\mathbb{R}^{m}$ с нормой

$$
\left\|\left(x_{1}, \ldots, x_{m}\right)\right\|_{q} \equiv\left\|\left(x_{1}, \ldots, x_{m}\right)\right\|_{l_{q}^{m}}= \begin{cases}\left(\left|x_{1}\right|^{q}+\cdots+\left|x_{m}\right|^{q}\right)^{\frac{1}{q}}, & \text { если } q<\infty, \\ \max \left\{\left|x_{1}\right|, \ldots,\left|x_{m}\right|\right\}, & \text { если } q=\infty,\end{cases}
$$

$B_{p}^{m}-$ единичный шар в $l_{p}^{m}$. 
Обозначим $\vartheta_{l}(M, X)=d_{l}(M, X)$ и $\widehat{q}=q$ при оценке колмогоровских поперечников, $\vartheta_{l}(M, X)=\lambda_{l}(M, X)$ и $\widehat{q}=\min \left\{q, p^{\prime}\right\}$ при оценке линейных поперечников, $\vartheta_{l}(M, X)=d^{l}(M, X)$ и $\widehat{q}=p^{\prime}$ при оценке гельфандовских поперечников.

Сформулируем результат об оценках поперечников $\vartheta_{n}\left(W_{p}^{r}\left([0,1]^{d}\right), L_{q}\left([0,1]^{d}\right)\right)$ при $p \leqslant q$ (см., например, [32]-[34], [36]).

Всюду далее полагаем $\delta=r+\frac{d}{q}-\frac{d}{p}$.

Будем использовать следующие обозначения для порядковых неравенств. Пусть $X, Y$ - множества, $f_{1}, f_{2}: X \times Y \rightarrow \mathbb{R}_{+}$. Обозначим

$$
f_{1}(x, y) \underset{y}{\lesssim} f_{2}(x, y)
$$

(или $\left.f_{2}(x, y) \underset{y}{\gtrsim} f_{1}(x, y)\right)$, если для любого $y \in Y$ существует $c(y)>0$ такое, что $f_{1}(x, y) \leqslant c(y) f_{2}(x, y)$ для любого $x \in X$;

$$
f_{1}(x, y) \underset{y}{\asymp} f_{2}(x, y),
$$

если $f_{1}(x, y) \underset{y}{\lesssim} f_{2}(x, y)$ и $f_{2}(x, y) \underset{y}{\lesssim} f_{1}(x, y)$.

Teоpema A (см. [32]-[34], [36]). Пусть $r \in \mathbb{N}, 1 \leqslant p \leqslant q \leqslant \infty, \delta>0$. Положим

$\theta_{p, q, r, d}= \begin{cases}\frac{\delta}{d}, & \text { если } p=q \text { или } p<q, \widehat{q} \leqslant 2, \\ \min \left\{\frac{\delta}{d}+\min \left\{\frac{1}{2}-\frac{1}{\widehat{q}}, \frac{1}{p}-\frac{1}{q}\right\}, \frac{\widehat{q} \delta}{2 d}\right\}, & \text { если } p<q, \widehat{q}>2 .\end{cases}$

Кроме того, предположим, что

$$
\frac{\delta}{d}+\min \left\{\frac{1}{2}-\frac{1}{\widehat{q}}, \frac{1}{p}-\frac{1}{q}\right\} \neq \frac{\widehat{q} \delta}{2 d}
$$

в случае $p<q, \widehat{q}>2$. Тогда

$$
\vartheta_{n}\left(W_{p}^{r}\left([0,1]^{d}\right), L_{q}\left([0,1]^{d}\right)\right) \underset{r, \widehat{d, p, q}}{\asymp} n^{-\theta_{p, q, r, d}} .
$$

Такие же оценки выполнены, если вместо куба берется область с липшицевой границей.

О.В. Бесов в статье [7] доказал результат о совпадении порядков поперечников

$$
d_{n}\left(W_{p}^{r}\left(K_{\sigma}\right), L_{q}\left(K_{\sigma}\right)\right) \underset{p, q, r, d, \sigma}{\asymp} d_{n}\left(W_{p}^{r}\left([0,1]^{d}\right), L_{q}\left([0,1]^{d}\right)\right),
$$

где

$$
\begin{gathered}
K_{\sigma}=\left\{\left(x_{1}, \ldots, x_{d-1}, x_{d}\right):\left|\left(x_{1}, \ldots, x_{d-1}\right)\right|^{\frac{1}{\sigma}}<x_{d}<1\right\}, \\
\sigma>1, \quad r-[\sigma(d-1)+1]\left(\frac{1}{p}-\frac{1}{q}\right)_{+}>0
\end{gathered}
$$

и в случае $p<q, \widehat{q}>2$ выполнено (1.2). Для $r=1, p=q$ и более общих нерегулярных областей оценки аппроксимативных чисел были получены В. Д. Эвансом и Д. Дж. Харрисом в [19]. 
Также изучались задача об оценке поперечников единичных шаров весовых пространств Соболева и других функциональных пространств и задача об оценке строгих $s$-чисел соответствующего оператора вложения. Оценка сверху колмогоровских поперечников единичных шаров пространств Соболева на кубе в весовом пространстве $L_{q}$ впервые была получена М.Ш. Бирманом и М. З. Соломяком (см. [37]) (при $q>\max \{p, 2\}$ оценка не была точной по порядку). Эль Колли в работе [38] нашел порядковые оценки величин $d_{n}\left(W_{p, g}^{r}(\Omega), L_{q, v}(\Omega)\right)$, где $\Omega$ - ограниченная область с гладкой границей, $p=q$, и веса $g$ и $v$ равны степени расстояния до границы $\Omega$; используя интерполяцию банаховых пространств, Х. Трибель (см. [8]) расширил верхние оценки поперечников $d_{n}\left(W_{p, g}^{r}(\Omega), L_{q, v}(\Omega)\right)$ на случай $p \leqslant q$. Для пересечений шаров некоторых пространств Соболева на кубе с весами, являющимися степенью расстояния до границы, порядковые оценки поперечников были получены И. В. Бойковым (см. [39], [40]). В [41] Х. Трибель получил оценки аппроксимативных чисел операторов вложения весовых пространств Соболева с весами, имеющими особенность в точке; этот результат был обобщен в статье [42]. Для весов общего вида колмогоровские и аппроксимативные числа оператора вложения весового пространства Соболева в весовое пространство $L_{q}$ были получены П. И. Лизоркиным, М. О. Отелбаевым, М. С. Айтеновой и Л. К. Кусаиновой (см. [43]-[46]); здесь в определение весового пространства Соболева входило ограничение не только на норму $r$-й производной, но и на норму самой функции, которое существенно использовалось.

Дадим определение области с условием Джона.

Обозначим через $A C\left[t_{0}, t_{1}\right]$ пространство абсолютно непрерывных функций на интервале $\left[t_{0}, t_{1}\right]$, а через $B_{a}(x)$ (соответственно $\left.\stackrel{\circ}{B}_{a}(x)\right)$ - замкнутый (соответственно открытый) евклидов шар в $\mathbb{R}^{d}$ радиуса $a$ с центром в точке $x$.

ОПредЕлЕниЕ 1. Пусть $G \subset \mathbb{R}^{d}$ - ограниченная область, $a>0, x_{*} \in G$. Скажем, что $G \in \mathbf{F C}\left(a, x_{*}\right)$, если для любого $x \in G$ существуют $T(x)>0$ и кривая $\gamma_{x}:[0, T(x)] \rightarrow G$ со следующими свойствами:

1) $\gamma_{x} \in A C[0, T(x)],\left|\frac{d \gamma_{x}(t)}{d t}\right|=1$ п.в.,

2) $\gamma_{x}(0)=x, \gamma_{x}(T(x))=x_{*}$,

3) $B_{a t}\left(\gamma_{x}(t)\right) \subset G$ для любого $t \in[0, T(x)]$.

Будем обозначать $G \in \mathbf{F C}(a)$, если $G \in \mathbf{F C}\left(a, x_{*}\right)$ для некоторого $x_{*} \in G$. Если $G \in \mathbf{F C}(a)$ для некоторого $a>0$, то будем говорить, что $G$ удовлетворяет условию Джона.

Для ограниченной области условие Джона эквивалентно условию гибкого конуса (см. определение в [47]). Примерами таких областей являются области с липшицевой границей, снежинка Коха, области вида $\bigcup_{0<t \leqslant T} \stackrel{\circ}{B}_{c t}(\gamma(t))$, где $\gamma:[0, T] \rightarrow \mathbb{R}^{d}-$ кривая с натуральной параметризацией, $c>0$. Области, имеющие нулевые внутренние углы, условию Джона не удовлетворяют.

Сформулируем основной результат статьи.

Всюду будем обозначать $\log t=\log _{2} t$.

Пусть $\Omega=D_{\varphi, G}$, где $G \subset \mathbb{R}^{d-1}, G \in \mathbf{F C}(a, 0)$. Будем рассматривать веса вида $g(y, z)=g_{0}(z), v(y, z)=v_{0}(z), \frac{y}{\varphi(z)} \in G, 0<z<\frac{1}{2}$. Предположим, что 
для любого $0<z \leqslant \frac{1}{2}$

$$
\begin{gathered}
g_{0}(z)=z^{-\beta_{g}}|\log z|^{-\alpha_{g}} \rho_{g}(|\log z|), \quad v_{0}(z)=z^{-\beta_{v}}|\log z|^{-\alpha_{v}} \rho_{v}(|\log z|), \\
\varphi(z)=z^{\sigma}|\log z|^{\theta} \omega(|\log z|),
\end{gathered}
$$

где $\rho_{g}, \rho_{v}, \omega$ - абсолютно непрерывные функции такие, что

$$
\begin{gathered}
\lim _{t \rightarrow+\infty} \frac{t \rho_{g}^{\prime}(t)}{\rho_{g}(t)}=\lim _{t \rightarrow+\infty} \frac{t \rho_{v}^{\prime}(t)}{\rho_{v}(t)}=\lim _{t \rightarrow+\infty} \frac{t \omega^{\prime}(t)}{\omega(t)}=0, \\
\sigma>1, \quad r+(\sigma(d-1)+1)\left(\frac{1}{q}-\frac{1}{p}\right)=\beta_{g}+\beta_{v}, \quad \sigma(d-1)+1-\beta_{v} q>0, \\
\alpha:=\alpha_{g}+\alpha_{v}+\theta(d-1)\left(\frac{1}{p}-\frac{1}{q}\right)>0 .
\end{gathered}
$$

Для таких весов $g, v$ и такой области $\Omega$ получены порядки поперечников множества $W_{p, g}^{r}(\Omega)$ в пространстве $L_{q, v}(\Omega)$.

Положим

$$
\rho(s):=\rho_{g}(s) \rho_{v}(s)[\omega(s)]^{(d-1)\left(\frac{1}{q}-\frac{1}{p}\right)},
$$

$\mathfrak{Z}:=(p, q, r, d, a, \varphi, g, v)$. Заметим, что $\lim _{t \rightarrow+\infty} \frac{t \rho^{\prime}(t)}{\rho(t)}=0$ и функция $t^{-\alpha} \rho(t)$ убывает при достаточно больших $t>0$.

Теорема 1. 1. Пусть $p=q$ или $p<q, \widehat{q} \leqslant 2, \alpha \neq \frac{\delta}{d}$. Положим $\sigma_{*}=0$, если $\alpha>\frac{\delta}{d}, u \sigma_{*}=1$, если $\alpha<\frac{\delta}{d}$. Тогда

$$
\vartheta_{n}\left(W_{p, g}^{r}(\Omega), L_{q, v}(\Omega)\right) \underset{\Im}{\Im} n^{-\min \left\{\frac{\delta}{d}, \alpha\right\}} \rho\left(n^{\sigma_{*}}\right) .
$$

2. Пусть $p<q u \widehat{q}>2$. Положим

$$
\begin{array}{cc}
\theta_{1}=\frac{\delta}{d}+\min \left\{\frac{1}{2}-\frac{1}{\widehat{q}}, \frac{1}{p}-\frac{1}{q}\right\}, & \theta_{2}=\frac{\widehat{q} \delta}{2 d}, \\
\theta_{3}=\alpha+\min \left\{\frac{1}{2}-\frac{1}{\widehat{q}}, \frac{1}{p}-\frac{1}{q}\right\}, & \theta_{4}=\frac{\widehat{q} \alpha}{2}, \\
\sigma_{1}=\sigma_{2}=0, \quad \sigma_{3}=1, & \sigma_{4}=\frac{\widehat{q}}{2} .
\end{array}
$$

Пусть существует $j_{*} \in\{1,2,3,4\}$ такое, что $\theta_{j_{*}}<\min _{j \neq j_{*}} \theta_{j}$. Тогда

$$
\vartheta_{n}\left(W_{p, g}^{r}(\Omega), L_{q, v}(\Omega)\right) \underset{3}{\Im} n^{-\theta_{j_{*}}} \rho\left(n^{\sigma_{j_{*}}}\right) .
$$

Если $\alpha>\frac{\delta}{d}$, то из теоремы А следует, что порядки поперечников такие же, как в случае $\Omega=[0,1]^{d}$ и единичных весов. При $\alpha<\frac{\delta}{d}$ оценки меняются. В частности, если

$$
\begin{aligned}
& g \equiv 1, \quad v \equiv 1, \\
& r+(\sigma(d-1)+1)\left(\frac{1}{q}-\frac{1}{p}\right)=0, \quad 0<\theta(d-1)\left(\frac{1}{p}-\frac{1}{q}\right)<\frac{\delta}{d},
\end{aligned}
$$

то порядки поперечников не такие, как в случае $\Omega=[0,1]^{d}$. 
Структура работы следующая. В $§ 2$ сформулированы известные результаты, которые будут применяться в дальнейшем. В 33 доказано обобщение результата В. Г. Мазьи и С. В. Поборчего (см. [6]) о непрерывном вложении на случай весовых пространств (при дополнительном условии $0 \in G$ ). В $\S 4$ доказана теорема 1 (а также ее обобщение для более сложных областей с пиком).

\section{§ 2. Предварительные сведения}

Для доказательства теоремы вложения весового пространства Соболева на области с пиком нам понадобится критерий ограниченности двухвесового оператора интегрирования на отрезке.

Пусть $t_{0}<t_{1}, r>0$, и пусть $u, w:\left[t_{0}, t_{1}\right] \rightarrow \mathbb{R}_{+}-$измеримые функции. Положим

$$
\widetilde{I}_{r, u, w, t_{1}} f(t)=w(t) \int_{t}^{t_{1}}(s-t)^{r-1} u(s) f(s) d s .
$$

Критерий непрерывности оператора $\widetilde{I}_{r, u, w, t_{1}}: L_{p}\left[t_{0}, t_{1}\right] \rightarrow L_{q}\left[t_{0}, t_{1}\right]$ доказан В. Д. Степановым (см. [48]). Сформулируем этот результат для $p \leqslant q$.

Teорема В (см. [48]). Пусть $r \geqslant 1,1<p \leqslant q<\infty$. Тогда

$$
\left\|\widetilde{I}_{r, u, w, t_{1}}\right\|_{L_{p} \rightarrow L_{q}} \underset{p, q, r}{\asymp} B_{0}+B_{1}
$$

где

$$
\begin{aligned}
& B_{0}=\sup _{t \in\left(t_{0}, t_{1}\right)}\left(\int_{t_{0}}^{t}(t-x)^{q(r-1)} w^{q}(x) d x\right)^{\frac{1}{q}}\left(\int_{t}^{t_{1}} u^{\frac{p}{p-1}}(x) d x\right)^{1-\frac{1}{p}}, \\
& B_{1}=\sup _{t \in\left(t_{0}, t_{1}\right)}\left(\int_{t_{0}}^{t} w^{q}(x) d x\right)^{\frac{1}{q}}\left(\int_{t}^{t_{1}}(x-t)^{\frac{p}{p-1}(r-1)} u^{\frac{p}{p-1}}(x) d x\right)^{1-\frac{1}{p}} .
\end{aligned}
$$

Обозначим через mes $\Omega$ меру Лебега множества $\Omega \subset \mathbb{R}^{d}$.

Пусть $\mathscr{P}_{r-1}\left(\mathbb{R}^{d}\right)$ - пространство полиномов на $\mathbb{R}^{d}$ степени не выше $r-1$. Для каждого измеримого подмножества $E \subset \mathbb{R}^{d}$ положим

$$
\mathscr{P}_{r-1}(E)=\left\{\left.f\right|_{E}: f \in \mathscr{P}_{r-1}\left(\mathbb{R}^{d}\right)\right\} .
$$

Ю. Г. Решетняк (см. [15], [16]) построил интегральное представление для гладких функций на области $\Omega$, удовлетворяющей условию Джона, в терминах их производных порядка $r$. Отсюда и из результатов С.Л. Соболева (см. [49], [50]) получаем следующую теорему.

Teоpema C (см. [15], [16], [49], [50]). Пустъ $\Omega \in \mathbf{F C}\left(a, x_{*}\right), r \in \mathbb{N}, 1<p \leqslant$ $q<\infty, \frac{r}{d}+\frac{1}{q}-\frac{1}{p} \geqslant 0$. Тогда существует линейный непрерывный оператор $P: L_{q}(\Omega) \rightarrow \mathscr{P}_{r-1}(\Omega)$ такой, что для любой функиии $f \in \mathscr{W}_{p}^{r}(\Omega)$

$$
\|f-P f\|_{L_{q}(\Omega)} \underset{p, q, r, d, a}{\lesssim}(\operatorname{mes} \Omega)^{\frac{\delta}{d}}\left\|\nabla^{r} f\right\|_{L_{p}(\Omega)}
$$

кроме того,

$$
\|f\|_{L_{q}(\Omega)} \underset{p, q, r, d, a}{\lesssim}(\operatorname{mes} \Omega)^{\frac{\delta}{d}}\left\|\nabla^{r} f\right\|_{L_{p}(\Omega)}+(\operatorname{mes} \Omega)^{\frac{1}{q}-\frac{1}{p}}\|f\|_{L_{p}(\Omega)},
$$




$$
\begin{gathered}
\|f\|_{L_{q}(\Omega)} \underset{p, q, r, d, a, c}{\lesssim}(\operatorname{mes} \Omega)^{\frac{\delta}{d}}\left\|\nabla^{r} f\right\|_{L_{p}(\Omega)}+\|f\|_{L_{q}\left(B_{c R}\left(x_{*}\right) \cap \Omega\right)}, \\
c>0, \quad R=\operatorname{diam} \Omega .
\end{gathered}
$$

Для доказательства оценок поперечников множеств $W_{p, g}^{r}(\Omega)$ нам понадобятся оценки поперечников конечномерных шаров $\vartheta_{n}\left(B_{p}^{\nu}, l_{q}^{\nu}\right)$, полученные Б. С. Кашиным и Е. Д. Глускиным (см. [34], [35]).

TeOpema D (см. [34], [35]). Пусть $1<p<q<\infty$. Тогда

$$
\begin{aligned}
& d_{n}\left(B_{p}^{\nu}, l_{q}^{\nu}\right) \underset{q, p}{\asymp} \Phi(n, \nu, p, q), \\
& \lambda_{n}\left(B_{p}^{\nu}, l_{q}^{\nu}\right) \underset{q, p}{\asymp} \Psi(n, \nu, p, q), \\
& d^{n}\left(B_{p}^{\nu}, l_{q}^{\nu}\right) \underset{q, p}{\asymp} \Phi\left(n, \nu, q^{\prime}, p^{\prime}\right),
\end{aligned}
$$

əəe

$$
\begin{gathered}
\Phi(n, \nu, p, q)= \begin{cases}\min \left\{1,\left(\nu^{\frac{1}{q}} n^{-\frac{1}{2}}\right)^{\left(\frac{1}{p}-\frac{1}{q}\right) /\left(\frac{1}{2}-\frac{1}{q}\right)}\right\}, & 2 \leqslant p<q<\infty, \\
\max \left\{\nu^{\frac{1}{q}-\frac{1}{p}}, \min \left(1, \nu^{\frac{1}{q}} n^{-\frac{1}{2}}\right)\left(1-\frac{n}{\nu}\right)^{\frac{1}{2}}\right\}, & 1<p<2<q<\infty, \\
\max \left\{\nu^{\frac{1}{q}-\frac{1}{p}},\left(1-\frac{n}{\nu}\right)^{\left(\frac{1}{q}-\frac{1}{p}\right) /\left(1-\frac{2}{p}\right)}\right\}, & 1<p<q \leqslant 2,\end{cases} \\
\Psi(n, \nu, p, q)= \begin{cases}\Phi(n, \nu, p, q), & \text { если } q \leqslant p^{\prime}, \\
\Phi\left(n, \nu, q^{\prime}, p^{\prime}\right), & \text { если } p^{\prime}<q .\end{cases}
\end{gathered}
$$

Если $p=q$, то

$$
\vartheta_{n}\left(B_{p}^{\nu}, l_{p}^{\nu}\right)=1 \quad \text { при } \quad n<\nu
$$

(см., например, [29; стр. 232], [30]).

Следующее утверждение было доказано в работе [51] (см. формулу (60)).

Лемма 1. Пусть $\Lambda_{*}:(0,+\infty) \rightarrow(0,+\infty)$ - абсолютно непрерывная функчия такая, что

$$
\lim _{y \rightarrow+\infty} \frac{y \Lambda_{*}^{\prime}(y)}{\Lambda_{*}(y)}=0 .
$$

Тогда для любого $\varepsilon>0$

$$
t^{-\varepsilon} \underset{\varepsilon, \Lambda_{*}}{\lesssim} \frac{\Lambda_{*}(t y)}{\Lambda_{*}(y)} \underset{\varepsilon, \Lambda_{*}}{\lesssim} t^{\varepsilon}, \quad 1 \leqslant y<\infty, \quad 1 \leqslant t<\infty .
$$

Повторим доказательство для оценки сверху (оценка снизу доказывается аналогично). Из (2.5) следует, что найдется $y_{\varepsilon}$ такое, что функция $y^{-\varepsilon} \Lambda_{*}(y)$ убывает на $\left[y_{\varepsilon}, \infty\right)$. Значит, если $y \geqslant y_{\varepsilon}, t \geqslant 1$, то

$$
\frac{\Lambda_{*}(t y)}{\Lambda_{*}(y)}=t^{\varepsilon} \frac{(t y)^{-\varepsilon} \Lambda_{*}(t y)}{y^{-\varepsilon} \Lambda_{*}(y)} \leqslant t^{\varepsilon} .
$$

Так как функция $\Lambda_{*}$ непрерывна, то найдется $C_{\varepsilon}>0$ такое, что $\frac{\Lambda_{*}(x)}{\Lambda_{*}(y)} \leqslant C_{\varepsilon}$ при $x, y \in\left[1, y_{\varepsilon}\right]$. Если $y \in\left[1, y_{\varepsilon}\right], t y>y_{\varepsilon}$, то

$$
\frac{\Lambda_{*}(t y)}{\Lambda_{*}(y)} \leqslant C_{\varepsilon} \frac{\Lambda_{*}(t y)}{\Lambda_{*}\left(y_{\varepsilon}\right)} \leqslant C_{\varepsilon}\left(\frac{t y}{y_{\varepsilon}}\right)^{\varepsilon} \leqslant C_{\varepsilon} t^{\varepsilon} .
$$




\section{§ 3. Теорема вложения для весового класса Соболева на области с пиком}

Для $y \in \mathbb{R}^{d-1}$ обозначим через $B_{a}^{d-1}(y)$ замкнутый евклидов шар в $\mathbb{R}^{d-1}$ радиуса $a$ с центром в точке $y$. В частности, положим $B^{d-1}=B_{1}^{d-1}(0)$.

Для $G \subset \mathbb{R}^{d}, x_{*} \in G$ обозначим

$$
\bar{R}_{x_{*}}(G)=\sup _{x \in G}\left\|x-x_{*}\right\|_{l_{2}^{d}}, \quad \underline{R}_{x_{*}}(G)=\inf _{x \in \partial G}\left\|x-x_{*}\right\|_{l_{2}^{d}}
$$

(здесь $\partial G$ - граница множества $G)$. Заметим, что если $G \in \mathbf{F C}\left(a, x_{*}\right)$, то

$$
\bar{R}_{x_{*}}(G) \underset{a, d}{\asymp} \underline{R}_{x_{*}}(G) .
$$

Для $z \in \mathbb{R}$ положим $\eta_{z}=(0, \ldots, 0, z) \in \mathbb{R}^{d}$.

Пусть $\varphi:(0,1) \rightarrow(0, \infty)$ - неубывающая липшицева функция такая, что $\lim _{z \rightarrow+0} \varphi(z)=\lim _{z \rightarrow+0} \varphi^{\prime}(z)=0$.

ОПРЕДЕЛЕНИЕ 2. Пусть $a>0, \tau_{*}>0$. Скажем, что $\Omega \in \mathbf{F C}_{\varphi, \tau_{*}}(a)$, если $\Omega=\bigcup_{z \in\left(0, \tau_{*}\right]} \Omega_{z}$, где $\Omega_{z} \in \mathbf{F C}\left(a, \eta_{z}\right)$,

$$
\begin{gathered}
\kappa_{\Omega}:=\sup _{z \in(0,1)} \frac{\bar{R}_{\eta_{z}}\left(\Omega_{z}\right)}{z}<1, \\
\underline{c} \varphi(z) \leqslant \bar{R}_{\eta_{z}}\left(\Omega_{z}\right) \leqslant \bar{c} \varphi(z), \quad z \in\left(0, \tau_{*}\right],
\end{gathered}
$$

для некоторых $0<\underline{c}<\bar{c}<\infty$.

Теоремы вложения и оценки поперечников будут доказаны для областей $\Omega \in$ $\mathbf{F C}_{\varphi, \tau_{*}}(a)$. В силу предложения 1 (см. ниже) те же результаты будут верны для областей вида $D_{\varphi, G}$ с $G \subset \mathbb{R}^{d-1}, G \in \mathbf{F C}(a, 0)$.

ПредлоЖение 1. Если $G \subset \mathbb{R}^{d-1}, G \in \mathbf{F C}(a, 0)$, а область $D_{\varphi, G} \subset \mathbb{R}^{d}$ определена формулой (1.1), то существует линейный изоморфизм $A: \mathbb{R}^{d} \rightarrow \mathbb{R}^{d}$ такой, что $A\left(D_{\varphi, G}\right) \in \mathbf{F C}_{\varphi, \tau_{*}}(b)$ для некоторых $b>0, \tau_{*}>0$.

ДоКАЗАТЕЛЬСтво. Положим $\mu_{0}=\operatorname{diam} G$. Из монотонности функции $\varphi$ следует, что существует единственное $\tau_{*} \in\left(0, \frac{1}{2}\right)$ такое, что $\tau_{*}+\mu_{0} \varphi\left(\tau_{*}\right)=\frac{1}{2}$. Для $0<z \leqslant \tau_{*}$ положим

$$
\Omega_{z}=\left\{(y, \zeta): z-\mu_{0} \varphi(z)<\zeta<z+\mu_{0} \varphi(z), y \in \varphi(\zeta) G\right\} .
$$

Так как функция $\varphi$ липшицева, то при достаточно малом $\mu_{0}$ выполнено (3.2) и

$$
z-\mu_{0} \varphi(z)>\frac{z}{2}, \quad z \in\left(0, \tau_{*}\right]
$$

Сделав гомотетическое преобразование переменных $y$, можно считать, что эти свойства выполнены. Кроме того, $\Omega_{z}$ удовлетворяет условию (3.3).

Положим

$$
D_{z}=\left\{(y, \zeta): z-\mu_{0} \varphi(z)<\zeta<z+\mu_{0} \varphi(z), y \in \varphi(z) G\right\} .
$$

Покажем, что существует такое $\widetilde{b}=\widetilde{b}\left(a, d, \mu_{0}, \varphi\right)>0$, что $D_{z} \in \mathbf{F C}\left(\widetilde{b}, \eta_{z}\right)$. 
В самом деле, пусть $y \in G, z-\mu_{0} \varphi(z)<\zeta<z+\mu_{0} \varphi(z), x=(\varphi(z) y, \zeta)$. В силу (3.1) существует такое $c=c(a, d)>0$, что $B_{c \mu_{0}}^{d-1}(0) \subset G$. Если $y \in$ $B_{c \mu_{0}}^{d-1}(0)$, то в качестве $\gamma_{x}$ из определения 1 берем натуральную параметризацию отрезка $\left[x, \eta_{z}\right]$. Пусть $y \notin B_{c \mu_{0}}^{d-1}(0), \widetilde{\gamma}_{y}:[0, T(y)] \rightarrow G-$ кривая со свойствами $1-3$ из определения 1 для области $G$. Тогда $T(y) \geqslant c \mu_{0}$. Определяем кривую $\gamma_{x}^{*}:[0, \varphi(z) T(y)] \rightarrow D_{z}$ по формуле

$$
\gamma_{x}^{*}(t)=\left(\varphi(z) \widetilde{\gamma}_{y}\left(\frac{t}{\varphi(z)}\right),\left(1-\frac{t}{\varphi(z) T(y)}\right) \zeta+\frac{t}{\varphi(z) T(y)} z\right) .
$$

Так как $\left|\frac{d \widetilde{\gamma}_{y}}{d t}\right|=1$ п.в., $|z-\zeta| \leqslant \mu_{0} \varphi(z), T(y) \geqslant c \mu_{0}$, то

$$
\left|\frac{d \gamma_{x}^{*}}{d t}\right|=\sqrt{1+\frac{|\zeta-z|^{2}}{T^{2}(y) \varphi^{2}(z)}} \subset\left[1, \sqrt{1+c^{-2}}\right] .
$$

Сделав линейную замену переменной $t$, получим эквивалентную натуральную параметризацию, которую обозначим через $\gamma_{x}$.

Определим липшицев гомеоморфизм $\Psi_{z}: \Omega_{z} \rightarrow D_{z}$ по формуле

$$
\Psi_{z}(y, \zeta)=\left(\frac{\varphi(z)}{\varphi(\zeta)} y, \zeta\right), \quad z-\mu_{0} \varphi(z)<\zeta<z+\mu_{0} \varphi(z), \quad y \in \varphi(\zeta) G
$$

Так как функция $\varphi$ липшицева и неубывающая, $\varphi^{\prime}(s) \rightarrow 0$ при $s \rightarrow 0$ и выполнено $(3.4)$, то $\varphi(\zeta) \underset{\mu_{0}, \varphi}{\asymp} \varphi(z)$ при всех $z \in\left(0, \tau_{*}\right], z-\mu_{0} \varphi(z)<\zeta<z+\mu_{0} \varphi(z)$. Для $y \in \varphi(\zeta) G$ выполнено $|y| \leqslant \mu_{0} \varphi(\zeta)$. Вычислив матрицу Якоби преобразования $\Psi_{z}$ и его обратного, получаем, что

$$
\left\|\Psi_{z}^{\prime}\right\| \underset{d, \mu_{0}, \varphi}{\lesssim} 1, \quad\left\|\left(\Psi_{z}^{-1}\right)^{\prime}\right\| \underset{d, \mu_{0}, \varphi}{\lesssim} 1
$$

Отсюда следует, что $\Omega_{z} \in \mathbf{F C}\left(b, \eta_{z}\right)$ для некоторого $b=b\left(a, d, \mu_{0}, \varphi\right)$. Это завершает доказательство предложения 1 .

Без ограничения общности можно считать, что $\tau_{*}=\frac{1}{2}$. Всюду будем обозначать $\mathbf{F C}_{\varphi}(a)=\mathbf{F C}_{\varphi, 1 / 2}(a)$.

Пусть $g_{0}:(0, \infty) \rightarrow(0, \infty), v_{0}:(0, \infty) \rightarrow(0, \infty)$ - измеримые функции,

$$
\begin{gathered}
\left.g_{0}\right|_{[1 / 2, \infty)} \equiv \text { const, }\left.\quad v_{0}\right|_{[1 / 2, \infty)} \equiv \text { const, } \\
g, v: \Omega \rightarrow(0, \infty), \quad g(y, z)=g_{0}(z), \quad v(y, z)=v_{0}(z) .
\end{gathered}
$$

Кроме того, предположим, что существует $C_{*}>0$ такое, что

$$
\begin{gathered}
\frac{g_{0}(t)}{g_{0}(s)} \leqslant C_{*}, \quad \frac{v_{0}(t)}{v_{0}(s)} \leqslant C_{*}, \quad z \in\left(0, \frac{1}{2}\right], \\
t, s \in\left[\max \left\{\frac{z}{2}, z-\varphi(z)\right\}, z+\varphi(z)\right] .
\end{gathered}
$$

Заметим, что $\max \left\{\frac{z}{2}, z-\varphi(z)\right\}=z-\varphi(z)$ при достаточно малых $z$.

Пусть $1<p \leqslant q<\infty, r \in \mathbb{N}, \delta:=r+\frac{d}{q}-\frac{d}{p}>0$. Обозначим

$$
\mathfrak{Z}_{1}=\left(p, q, r, d, a, \varphi, \kappa_{\Omega}, \underline{c}, \bar{c}, C_{*}\right) .
$$


Для $x=\left(x_{1}, \ldots, x_{d}\right) \in \mathbb{R}^{d}$ положим $x^{\prime}=\left(x_{1}, \ldots, x_{d-1}\right)$.

Обозначим $\bar{R}_{z}=\bar{R}_{\eta_{z}}\left(\Omega_{z}\right), \underline{R}_{z}=\underline{R}_{\eta_{z}}\left(\Omega_{z}\right)$.

Для $0 \leqslant \tau_{-}<\tau_{+} \leqslant \frac{1}{2}$ положим $\Omega_{\left[\tau_{-}, \tau_{+}\right]}=\bigcup_{z \in\left[\tau_{-}, \tau_{+}\right]} \Omega_{z}\left(\right.$ с $\left.\Omega_{0}=\varnothing\right)$. Заметим, что $\Omega_{\left[\tau_{-}, \tau_{+}\right]}$является областью.

Следующая теорема является обобщением результата В. Г. Мазьи и С. В. Поборчего (см. [6]) о вложениях пространств Соболева на области с пиком на случай весов $g(y, z)=g_{0}(z), v(y, z)=v_{0}(z)$, удовлетворяющих (3.5).

Tеорема 2. Пустъ $\Omega \in \mathbf{F C}_{\varphi}(a), 0 \leqslant \tau_{-}<\tau_{+} \leqslant \frac{1}{2}$,

$$
\begin{gathered}
\tau_{-}<\tau_{+}-\underline{R}_{\tau_{+}}, \quad 0<\lambda<1, \quad R=\lambda \underline{R}_{\tau_{+}}, \\
\widehat{W}_{p, g ; R}^{r}\left(\Omega_{\left[\tau_{-}, \tau_{+}\right]}\right)=\left\{f \in W_{p, g}^{r}\left(\Omega_{\left[\tau_{-}, \tau_{+}\right]}\right):\left.f\right|_{B_{R}\left(\eta_{\tau_{+}}\right)}=0\right\} .
\end{gathered}
$$

Тогда множество $\widehat{W}_{p, g ; R}^{r}\left(\Omega_{\left[\tau_{-}, \tau_{+}\right]}\right)$ограничено $L_{q, v}\left(\Omega_{\left[\tau_{-}, \tau_{+}\right]}\right)$в том и только в том случае, если

$$
A_{\left[\tau_{-}, \tau_{+}\right]}:=\max \left\{A_{0,\left[\tau_{-}, \tau_{+}\right]}, A_{1,\left[\tau_{-}, \tau_{+}\right]}\right\}<\infty,
$$

əдe

$$
\begin{aligned}
& A_{0,\left[\tau_{-}, \tau_{+}\right]}=\sup _{t \in\left(\tau_{-}, \tau_{+}\right)}\left(\int_{\tau_{-}}^{t} \varphi^{d-1}(z) v_{0}^{q}(z) d z\right)^{\frac{1}{q}}\left(\int_{t}^{\tau_{+}}(z-t)^{p^{\prime}(r-1)} g_{0}^{p^{\prime}}(z) \varphi^{\frac{d-1}{1-p}}(z) d z\right)^{\frac{1}{p^{\prime}}}, \\
& A_{1,\left[\tau_{-}, \tau_{+}\right]}=\sup _{t \in\left(\tau_{-}, \tau_{+}\right)}\left(\int_{\tau_{-}}^{t}(t-z)^{q(r-1)} \varphi^{d-1}(z) v_{0}^{q}(z) d z\right)^{\frac{1}{q}}\left(\int_{t}^{\tau_{+}} g_{0}^{p^{\prime}}(z) \varphi^{\frac{d-1}{1-p}}(z) d z\right)^{\frac{1}{p^{\prime}}} .
\end{aligned}
$$

Кроме того, если $I: \operatorname{span} \widehat{W}_{p, g ; R}^{r}\left(\Omega_{\left[\tau_{-}, \tau_{+}\right]}\right) \rightarrow L_{q, v}\left(\Omega_{\left[\tau_{-}, \tau_{+}\right]}\right)$- оператор вложения, mo $\|I\| \underset{\mathfrak{Z}_{1}, \lambda}{\asymp} A_{\left[\tau_{-}, \tau_{+}\right]}$.

Лемма 2. Пусть $0<\tau_{0}<\tau_{1} \leqslant \frac{1}{2} u c>0$ maковы, ито

$$
\tau_{1}-\tau_{0} \leqslant c \varphi\left(\tau_{0}\right)
$$

u пусть $L$ - константа Липшица функции $\left.\varphi\right|_{\left[\tau_{0}, \tau_{1}\right]} \cdot$ Тогда $\bigcup_{z \in\left[\tau_{0}, \tau_{1}\right]} \Omega_{z} \in$ $\mathbf{F C}\left(b, \eta_{\tau}\right)$ для любого $\tau \in\left[\tau_{0}, \tau_{1}\right]$, где $b=b(a, d, c, L, \underline{c}, \bar{c})>0$.

ДокАЗАТЕльство. Для любого $t \in\left[\tau_{0}, \tau_{1}\right]$

$$
\varphi\left(\tau_{0}\right) \leqslant \varphi(t) \leqslant \varphi\left(\tau_{0}\right)+L\left(t-\tau_{0}\right) \leqslant(L \cdot c+1) \varphi\left(\tau_{0}\right) .
$$

Поэтому

$$
\underline{R}_{t} \underset{a, d}{\stackrel{(3.1)}{\widetilde{C}}} \bar{R}_{t} \underset{\underline{c}, \overline{\bar{c}, L, c}}{\stackrel{(3.3)}{\leftrightarrows}} \varphi\left(\tau_{0}\right) .
$$

Пусть $z \in\left[\tau_{0}, \tau_{1}\right], x \in \Omega_{z}, \gamma_{x}:[0, T(x)] \rightarrow \Omega_{z}-$ кривая из определения 1 , $\gamma_{x}(T(x))=\eta_{z}$. Тогда $T(x) \leqslant a^{-1} \underline{R}_{z}$. Продолжим кривую $\gamma_{x}$, соединив $\eta_{z}$ и $\eta_{\tau}$ отрезком. Остается применить (3.8) и (3.9). Лемма доказана.

Для $z \in\left(0, \frac{1}{2}\right]$ обозначим $G_{z}=\left\{y \in \mathbb{R}^{d-1}:(y, z) \in \Omega\right\}$. Тогда

$$
G_{z} \supset\left\{y \in \mathbb{R}^{d-1}:(y, z) \in \Omega_{z}\right\} \supset B_{\underline{R}_{z}}^{d-1}(0),
$$


откуда

$$
\operatorname{dist}\left(0, \partial G_{z}\right) \geqslant \underline{R}_{z}, \quad \operatorname{diam} G_{z} \geqslant 2 \underline{R}_{z} .
$$

Из (3.1) и (3.3) следует, что

$$
\underline{R}_{z} \underset{a, d}{\asymp} \bar{R}_{z} \geqslant \underline{c} \varphi(z) .
$$

ЛЕмма 3. Пусть

$$
\zeta \in(0,1), \quad \Omega_{\zeta} \cap\left\{(y, z): y \in \mathbb{R}^{d-1}\right\} \neq \varnothing .
$$

Тогда $\varphi(\zeta) \underset{\mathfrak{Z}_{1}}{\asymp} \varphi(z)$.

ДоказАтельство. Из (3.2) и (3.3) следует, что $\bar{R}_{\zeta} \leqslant \bar{c} \varphi(\zeta)$ и $\bar{R}_{\zeta} \leqslant \kappa_{\Omega} \zeta$. Далее, $|\zeta-z| \leqslant \bar{R}_{\zeta}$. Таким образом, $\zeta-\bar{c} \varphi(\zeta) \leqslant z \leqslant \zeta+\bar{c} \varphi(\zeta)$ и $\left(1-\kappa_{\Omega}\right) \zeta \leqslant$ $z \leqslant\left(1+\kappa_{\Omega}\right) \zeta$. Значит, $z=\zeta+\theta(\zeta) \varphi(\zeta)$, где $\theta(\zeta) \in[-\bar{c}, \bar{c}]$. Так как функция $\varphi$ липшицева, то

$$
\varphi(z)=\varphi(\zeta+\theta(\zeta) \varphi(\zeta))=\varphi(\zeta)+\int_{0}^{\theta(\zeta) \varphi(\zeta)} \varphi^{\prime}(\zeta+s) d s
$$

Напомним, что $\varphi^{\prime}(t) \underset{t \rightarrow+0}{\longrightarrow} 0$. Поэтому для любого $\varepsilon>0$ существует $t_{*}(\varepsilon)>0$ такое, что $\varphi^{\prime}(t)<\varepsilon$ для п.в. $t \in\left(0, t_{*}(\varepsilon)\right)$. Так как

$$
\zeta \leqslant \frac{z}{1-\kappa_{\Omega}}, \quad \varphi(t) \underset{t \rightarrow+0}{\longrightarrow} 0, \quad \varphi^{\prime}(t) \underset{t \rightarrow+0}{\longrightarrow} 0,
$$

то существует $z_{*}(\varepsilon)>0$ такое, что $\varphi^{\prime}(\zeta+s)<\varepsilon$ для $s \in[-\bar{c} \varphi(\zeta), \bar{c} \varphi(\zeta)], z \in$ $\left(0, z_{*}(\varepsilon)\right]$; в самом деле, $\zeta-\bar{c} \varphi(\zeta)>0$ при малых $z$ и

$$
\zeta+s \leqslant \frac{z}{1-\kappa_{\Omega}}+\bar{c} \varphi\left(\frac{z}{1-\kappa_{\Omega}}\right) \underset{z \rightarrow+0}{\longrightarrow} 0 .
$$

Значит, в силу (3.13)

$$
|\varphi(z)-\varphi(\zeta)| \leqslant \bar{c} \varepsilon \varphi(\zeta) .
$$

Возьмем $\varepsilon=\frac{1}{2 \bar{c}}$ и получим $\frac{\varphi(\zeta)}{2} \leqslant \varphi(z) \leqslant \frac{3 \varphi(\zeta)}{2}$ для любого $z \in\left(0, z_{*}\left(\frac{1}{2} \bar{c}\right)\right]$ и $\zeta$, удовлетворяющего (3.12). Для $z>z_{*}\left(\frac{1}{2} \bar{c}\right)$ используем соотношение $z \underset{\kappa_{\Omega}}{\asymp} \zeta$ и учитываем, что функция $\varphi$ неубывающая. Лемма доказана.

ЛЕмма 4. Выполнены соотношения

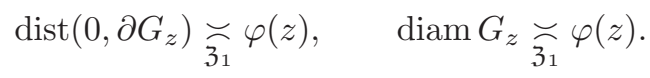

ДокАЗАТЕЛЬСтво. Оценки снизу следуют из (3.10) и (3.11). Оценки сверху следуют из определения 2 и леммы 3.

Лемма 5. Пусть функиии $g_{0}, v_{0}$ удовлетворяют $(3.5), \lambda_{*} \geqslant 1$. Тогда

$$
g_{0}(t) \underset{\mathfrak{Z}_{1}, \lambda_{*}}{\asymp} g_{0}(z), \quad v_{0}(t) \underset{\mathfrak{J}_{1}, \lambda_{*}}{\asymp} v_{0}(z)
$$

для любого $z \in\left(0, \frac{1}{2}\right]$ и любого

$$
t \in\left[\max \left\{\frac{z}{2 \lambda_{*}}, z-\lambda_{*} \varphi(z)\right\}, \min \left\{z+\lambda_{*} \varphi(z), \frac{1}{2}\right\}\right] .
$$


ДокАзАтельство. Сначала покажем, что для $\lambda_{*} \geqslant 1$

$g_{0}(t) \underset{\mathfrak{Z}_{1}, \lambda_{*}}{\asymp} g_{0}(z), \quad v_{0}(t) \underset{\mathfrak{Z}_{1}, \lambda_{*}}{\asymp} v_{0}(z), \quad 0<z \leqslant \frac{1}{2}, \quad z \leqslant t \leqslant \min \left\{z+\lambda_{*} \varphi(z), \frac{1}{2}\right\}$.

Для этого построим последовательность $\left\{z_{(k)}\right\}$ по индукции. Положим $z_{(0)}=z$. Пусть построено $z_{(k)} \leqslant \frac{1}{2}$ для некоторого $k \in \mathbb{Z}_{+}$. Если $z_{(k)}=\frac{1}{2}$, то построение останавливаем. Если $z_{(k)}<\frac{1}{2}$, то полагаем $z_{(k+1)}=\min \left\{z_{(k)}+\varphi\left(z_{(k)}\right), \frac{1}{2}\right\}$. Пусть $k \in \mathbb{N}, z_{(k)}<\frac{1}{2}$. Так как функция $\varphi$ неубывающая, то $z_{(k)} \geqslant z_{(k-1)}+\varphi(z)$. Значит, $z_{(k)} \geqslant z+k \varphi(z)$, и для $k=\left\lceil\lambda_{*}\right\rceil$ получаем $z_{(k)} \geqslant z+\lambda_{*} \varphi(z)$. Остается заметить, что

$$
g_{0}(t) \underset{\mathfrak{Z}_{1}}{\asymp} g_{0}\left(z_{(j-1)}\right), \quad v_{0}(t) \underset{\mathfrak{Z}_{1}}{\asymp} v_{0}\left(z_{(j-1)}\right)
$$

для любого $t \in\left[z_{(j-1)}, z_{(j)}\right]$.

Пусть

$$
\max \left\{\frac{z}{2 \lambda_{*}}, z-\lambda_{*} \varphi(z)\right\} \leqslant t \leqslant z .
$$

Так как $\lim _{s \rightarrow+0} \varphi^{\prime}(s)=0$, то существует $z_{*}=z_{*}\left(\mathfrak{Z}_{1}, \lambda_{*}\right)$ такое, что $\frac{z}{2} \leqslant z-$ $\lambda_{*} \varphi(z)$ и $\left|\varphi^{\prime}(z)\right| \leqslant \frac{1}{2 \lambda_{*}}$ для любого $z \leqslant z_{*}$. Тогда для любого $z \leqslant z_{*}$ получаем $\varphi\left(z-\lambda_{*} \varphi(z)\right) \geqslant \frac{\varphi(z)}{2}$. Следовательно,

$$
z \leqslant z-\lambda_{*} \varphi(z)+2 \lambda_{*} \varphi\left(z-\lambda_{*} \varphi(z)\right) .
$$

Остается применить (3.15).

Пусть $z>z_{*}$. Докажем, что

$$
g_{0}(z) \underset{\mathfrak{Z}_{1}, \lambda_{*}}{\asymp} g_{0}(t), \quad v_{0}(z) \underset{\mathfrak{Z}_{1}, \lambda_{*}}{\asymp} v_{0}(t)
$$

для любого $t \in\left[\frac{z}{2 \lambda_{*}}, z\right]$. Имеем

$$
\varphi\left(\frac{z}{2 \lambda_{*}}\right) \geqslant \varphi\left(\frac{z_{*}}{2 \lambda_{*}}\right) \underset{3_{1}, \lambda_{*}}{\asymp} 1 .
$$

Это означает, что существует $c_{\mathfrak{Z}_{1}, \lambda_{*}}>0$ такое, что

$$
z \leqslant \frac{z}{2 \lambda_{*}}+c_{\mathfrak{Z}_{1}, \lambda_{*}} \varphi\left(\frac{z}{2 \lambda_{*}}\right) .
$$

Применив (3.15) еще раз, получаем требуемую оценку. Лемма 5 доказана.

Пусть $T$ - не более чем счетное семейство измеримых множеств. Положим

$$
\begin{gathered}
\mathscr{N}_{T, E}=\operatorname{card}\left\{E^{\prime} \in T: \operatorname{mes}\left(E \cap E^{\prime}\right)>0\right\}, \quad E \in T, \\
\mathscr{N}_{T}=\sup _{E \in T} \mathscr{N}_{T, E} .
\end{gathered}
$$

Лемма 6. Пусть $k \in \mathbb{N} \cup\{\infty\}, 0 \leqslant \tau_{k}<\cdots<\tau_{2}<\tau_{1}<\tau_{0} \leqslant \frac{1}{2}, 0<\widehat{c}<1$,

$$
\tau_{j-1}-\tau_{j} \geqslant \widehat{c} \varphi\left(\tau_{j-1}\right),
$$

$G_{(j)}=\Omega_{\left[\tau_{j}, \tau_{j-1}\right]}, 1 \leqslant j<k+1, T=\left\{G_{(j)}\right\}_{j=1}^{k}$. Тогда

$$
\mathscr{N}_{T} \lesssim 1
$$


ДоказАтельство. Так как функция $\varphi$ липшицева и $\lim _{z \rightarrow 0} \varphi^{\prime}(z)=0$, то существует $z_{0}=z_{0}\left(\mathfrak{Z}_{1}\right) \in\left(0, \frac{1}{2}\right]$ такое, что для любого $z \in\left(0, z_{0}\right]$

$$
\varphi(z-2 \bar{c} \varphi(z)) \geqslant \frac{\varphi(z)}{2}, \quad \varphi(z+2 \bar{c} \varphi(z)) \leqslant 2 \varphi(z), \quad\left|\varphi^{\prime}(z)\right| \leqslant \frac{1}{4 \bar{c}} .
$$

Оценим

$$
\operatorname{card}\left\{i \in \overline{1, k} \backslash\{j\}: G_{(i)} \cap G_{(j)} \neq \varnothing\right\}, \quad j \in \overline{1, k} .
$$

Так как множества $G_{(i)}$ открыты, то условие $G_{(i)} \cap G_{(j)} \neq \varnothing$ эквивалентно неравенству $\operatorname{mes}\left(G_{(i)} \cap G_{(j)}\right)>0$. Заметим, что если $\tau_{i-1} \geqslant z_{0}$, то

$$
\tau_{i-1}-\tau_{i} \stackrel{(3.16)}{\geqslant} \widehat{c} \varphi\left(\tau_{i-1}\right) \underset{3_{1}, \widehat{c}}{\asymp} 1 \text {. }
$$

Значит,

$$
\operatorname{card}\left\{i \in \overline{1, k}: \tau_{i-1} \geqslant z_{0}\right\} \underset{\mathfrak{Z}_{1}, \widehat{c}}{\lesssim} 1 .
$$

Поэтому достаточно оценить

$$
\operatorname{card}\left\{i \in \overline{1, k} \backslash\{j\}: G_{(i)} \cap G_{(j)} \neq \varnothing, \tau_{i-1}<z_{0}\right\} .
$$

Если $G_{(i)} \cap G_{(j)} \neq \varnothing$, то существуют $z=z_{i, j} \in\left[\tau_{j}, \tau_{j-1}\right], t \in\left[\tau_{i}, \tau_{i-1}\right]$ такие, что

$$
B_{\bar{R}_{z}}\left(\eta_{z}\right) \cap B_{\bar{R}_{t}}\left(\eta_{t}\right) \neq \varnothing .
$$

Пусть $i<j$. Тогда из (3.18) следует, что $z+\bar{R}_{z} \geqslant t-\bar{R}_{t}$. Поэтому

$$
t-\tau_{j-1} \leqslant t-z \leqslant \bar{R}_{z}+\bar{R}_{t} \stackrel{(3.3)}{\leqslant} \bar{c} \varphi(z)+\bar{c} \varphi(t) \leqslant 2 \bar{c} \varphi(t),
$$

т.е. $t-2 \bar{c} \varphi(t) \leqslant \tau_{j-1}$. В силу монотонности функции $\varphi$ и неравенства $t \leqslant \tau_{i-1} \leqslant$ $z_{0}$, выполнено

$$
\frac{\varphi(t)}{2} \stackrel{(3.17)}{\leqslant} \varphi(t-2 \bar{c} \varphi(t)) \leqslant \varphi\left(\tau_{j-1}\right) .
$$

Применяя еще раз (3.19), получаем $\tau_{i}-\tau_{j-1} \leqslant 2 \bar{c} \varphi(t) \leqslant 4 \bar{c} \varphi\left(\tau_{j-1}\right)$. С другой стороны,

$$
\tau_{i-1}-\tau_{i} \stackrel{(3.16)}{\geqslant} \widehat{c} \varphi\left(\tau_{i-1}\right) \geqslant \widehat{c} \varphi\left(\tau_{j-1}\right)
$$

Из последних двух цепочек неравенств получаем требуемую оценку.

Пусть $i>j$. Тогда из (3.18) следует, что

$$
t+\bar{R}_{t} \geqslant z-\bar{R}_{z}
$$

Пусть $z \geqslant z_{0}$. Тогда

$$
\left(1+\kappa_{\Omega}\right) \tau_{i-1} \geqslant\left(1+\kappa_{\Omega}\right) t \stackrel{(3.2)}{\geqslant} t+\bar{R}_{t} \stackrel{(3.20)}{\geqslant} z-\bar{R}_{z} \stackrel{(3.2)}{\geqslant}\left(1-\kappa_{\Omega}\right) z \geqslant\left(1-\kappa_{\Omega}\right) z_{0} .
$$

Значит, $\tau_{i-1} \underset{\mathfrak{Z}_{1}}{\gtrsim} 1, \varphi\left(\tau_{i-1}\right) \underset{\mathfrak{Z}_{1}}{\gtrsim} 1, \tau_{i-1}-\tau_{i} \underset{\mathfrak{Z}_{1}, \widehat{c}}{(3.16)} 1$ и

$$
\operatorname{card}\left\{i>j: G_{(i)} \cap G_{(j)} \neq \varnothing, z_{i, j} \geqslant z_{0}\right\} \underset{\mathfrak{Z}_{1}, \widehat{c}}{\lesssim} 1 .
$$


Пусть $z<z_{0}$. Из (3.20) следует, что

$$
z-\bar{R}_{z} \leqslant t+\bar{R}_{t} \stackrel{(3.3)}{\leqslant} t+\bar{c} \varphi(t) \leqslant \tau_{i-1}+\bar{c} \varphi(z)
$$

Положим

$$
\widetilde{z}= \begin{cases}z, & \text { если } z-\bar{R}_{z} \leqslant \tau_{j}-\bar{R}_{\tau_{j}}, \\ \tau_{j}, & \text { иначе. }\end{cases}
$$

Тогда

$$
\widetilde{z}-\bar{R}_{\widetilde{z}} \leqslant \tau_{i-1}+\bar{c} \varphi(\widetilde{z}) .
$$

В самом деле, если $\widetilde{z}=z$, то это следует из (3.21). Если $\widetilde{z}=\tau_{j}$, то в силу $(3.21),(3.22)$ и неравенств $t \leqslant \tau_{i-1} \leqslant \tau_{j}$ получаем

$$
\widetilde{z}-\bar{R}_{\widetilde{z}} \leqslant z-\bar{R}_{z} \leqslant t+\bar{c} \varphi(t) \leqslant \tau_{i-1}+\bar{c} \varphi\left(\tau_{j}\right)=\tau_{i-1}+\bar{c} \varphi(\widetilde{z}) .
$$

Оценим $\widetilde{z}-\tau_{j}$ сверху. Учитывая условие $\widetilde{z} \leqslant z<z_{0}$, имеем

$$
\begin{aligned}
\widetilde{z}-\tau_{j} & \stackrel{(3.22)}{\leqslant} \bar{R}_{\widetilde{z}}-\bar{R}_{\tau_{j}} \stackrel{(3.3)}{\leqslant} \bar{c} \varphi(\widetilde{z})-\underline{c} \varphi\left(\tau_{j}\right) \\
& =(\bar{c}-\underline{c}) \varphi\left(\tau_{j}\right)+\bar{c} \int_{\tau_{j}}^{\widetilde{z}} \varphi^{\prime}(s) d s \stackrel{(3.17)}{\leqslant}(\bar{c}-\underline{c}) \varphi\left(\tau_{j}\right)+\frac{\widetilde{z}-\tau_{j}}{4}
\end{aligned}
$$

откуда

$$
\widetilde{z}-\tau_{j} \leqslant 2(\bar{c}-\underline{c}) \varphi\left(\tau_{j}\right)
$$

Из (3.23) получаем

$$
\widetilde{z}-\tau_{i-1} \leqslant \bar{R}_{\widetilde{z}}+\bar{c} \varphi(\widetilde{z}) \stackrel{(3.3)}{\leqslant} 2 \bar{c} \varphi(\widetilde{z})
$$

Значит, $\varphi\left(\tau_{i-1}\right) \geqslant \varphi(\widetilde{z}-2 \bar{c} \varphi(\widetilde{z})) \stackrel{(3.17)}{\geqslant} \frac{\varphi(\widetilde{z})}{2}$. Следовательно,

$$
\tau_{i-1}-\tau_{i} \stackrel{(3.16)}{\geqslant} \widehat{c} \varphi\left(\tau_{i-1}\right) \geqslant \frac{\widehat{c}}{2} \varphi(\widetilde{z}) \geqslant \frac{\widehat{c}}{2} \varphi\left(\tau_{j}\right)
$$

С другой стороны,

$$
\tau_{j}-\tau_{i-1} \leqslant \widetilde{z}-\tau_{i-1} \stackrel{(3.25)}{\leqslant} 2 \bar{c} \varphi(\widetilde{z}) \stackrel{(3.17),(3.24)}{\leqslant} 4 \bar{c} \varphi\left(\tau_{j}\right) .
$$

Из последних двух цепочек неравенств получаем требуемую оценку. Лемма 6 доказана.

Следующее неравенство аналогично неравенству (4.7) в статье [6], если $\tau_{+}-$ $\tau_{-} \geqslant \lambda_{*} \varphi\left(\tau_{+}\right), \lambda_{*}>0$, то:

$$
\sup _{z \in\left[\tau_{-}, \tau_{+}\right]} g_{0}(z) v_{0}(z) \varphi(z)^{r+\frac{d}{q}-\frac{d}{p}} \underset{\mathfrak{Z}_{1}, \lambda_{*}}{\lesssim} A_{0,\left[\tau_{-}, \tau_{+}\right]}
$$

Докажем теорему 2. 
ДокАзАтЕльство. Рассуждения почти такие же, как в [6]. Приведем схему доказательства.

Для доказательства оценки снизу берем функции

$$
\psi_{f}(y, z)= \begin{cases}\int_{z}^{\tau_{+}-R}(t-z)^{r-1} g_{0}(t) f(t) d t, & z \leqslant \tau_{+}-R \\ 0, & z>\tau_{+}-R\end{cases}
$$

где $f$ такова, что функция $f_{*}(y, z)=f(z)$ удовлетворяет условию $\left\|f_{*}\right\|_{L_{p}(\Omega)}=1$ и $f(z)=0$ для $z \notin\left[\tau_{-}, \tau_{+}\right]$. По теореме В

$$
\sup _{\left\|f_{*}\right\|_{L_{p}(\Omega)}=1}\left\|\psi_{f}\right\|_{L_{q, v}(\Omega)} \underset{\mathfrak{Z}_{1}}{\gtrsim} \max \left\{A_{0,\left[\tau_{-}, \tau_{+}-R\right]}, A_{1,\left[\tau_{-}, \tau_{+}-R\right]}\right\} .
$$

Применяя лемму 5, учитывая липшицевость $\varphi$ и используя неравенства

$$
\begin{gathered}
R \leqslant \bar{R}_{\tau_{+}} \stackrel{(3.3)}{\leqslant} \bar{c} \varphi\left(\tau_{+}\right), \\
\tau_{+}-R-\tau_{-}=\tau_{+}-\underline{R}_{\tau_{+}}-\tau_{-}+\left(\underline{R}_{\tau_{+}}-R\right) \stackrel{(3.6)}{\geqslant} \underline{R}_{\tau_{+}}-R \stackrel{(3.6)}{=}\left(\frac{1}{\lambda}-1\right) R,
\end{gathered}
$$

получаем

$$
\max \left\{A_{0,\left[\tau_{-}, \tau_{+}-R\right]}, A_{1,\left[\tau_{-}, \tau_{+}-R\right]}\right\} \underset{\mathfrak{Z}_{1}, \lambda}{\gtrsim} \max \left\{A_{0,\left[\tau_{-}, \tau_{+}\right]}, A_{1,\left[\tau_{-}, \tau_{+}\right]}\right\} .
$$

Докажем оценку сверху. По лемме 2.2 из [6], можно считать, что

$$
\varphi \in C^{r}\left(0, \frac{1}{2}\right], \quad\left|\varphi^{(k)}(z)\right| \leqslant c \varphi(z)^{1-k}, \quad k \in \overline{1, r}, \quad z \in\left(0, \frac{1}{2}\right],
$$

для некоторого $c>0$, не зависящего от $z$. Из (3.10) и (3.11) следует, что существует $c_{*}=c_{*}\left(\mathfrak{Z}_{1}\right)>0$ такое, что $\operatorname{dist}\left(0, \partial G_{z}\right) \geqslant \underline{R}_{z} \geqslant c_{*} \varphi(z)$. Применив гомотетическое преобразование по переменной $y$, можем считать $\frac{\lambda c_{*}}{2}=1$. Пусть

$$
\begin{gathered}
K \in C_{0}^{\infty}\left(B^{d-1}\right), \quad \int_{B^{d-1}} K(y) d y=1, \quad \int_{B^{d-1}} K(y) y^{\mu} d y=0, \\
\mu \in \mathbb{Z}_{+}^{d-1}, \quad 1 \leqslant|\mu| \leqslant r-1, \\
u_{\mu}(z)=\varphi(z)^{1-d} \int_{|y|<\varphi(z)} K\left(\frac{y}{\varphi(z)}\right) \frac{\partial^{|\mu|} u}{\partial y^{\mu}}(y, z) d y, \quad z \in\left(0, \frac{1}{2}\right], \quad u \in W_{p, g}^{r}(\Omega), \\
Q(x)=\sum_{|\mu| \leqslant r-1} u_{\mu}(z) \frac{y^{\mu}}{\mu !}, \quad x=(y, z) \in \Omega
\end{gathered}
$$

(см. $[6 ; \S 4])$.

В [6; лемма 3.1] доказано, что если выполнено (3.27), то функция $u_{\mu}$ абсолютно непрерывна вместе с производными порядка не выше $r-1$. Кроме того, $u_{\mu}^{(r)} \in L_{p}^{\text {loc }}\left(0, \frac{1}{2}\right)$ и для $r-|\mu| \leqslant s \leqslant r, z \in\left(0, \frac{1}{2}\right)$, выполнено

$$
\left|u_{\mu}^{(s)}(z)\right| \underset{\mathfrak{Z}_{1}}{\lesssim} \varphi(z)^{r-|\mu|-s-\frac{d-1}{p}}\left(\int_{|y|<\varphi(z)}\left|\nabla^{r} u(y, z)\right|^{p} d y\right)^{\frac{1}{p}} .
$$


Положим $\widetilde{\Omega}_{\left[\tau_{-}, \tau_{+}\right]}=\left\{(y, z) \in \Omega_{\left[\tau_{-}, \tau_{+}\right]}: \tau_{-} \leqslant z \leqslant \tau_{+}\right\}$.

Пусть

$$
\mu \in \mathbb{Z}_{+}^{d-1}, \quad|\mu| \leqslant r-1, \quad Q_{\mu}(x)=u_{\mu}(z) y^{\mu} .
$$

Оценим сверху $\left\|Q_{\mu}\right\|_{L_{q, v}\left(\widetilde{\Omega}_{\left[\tau_{-}, \tau_{+}\right]}\right)}$. Так как $u_{\mu}(z)=0$ в некоторой окрестности $\tau_{+}$ (см. (3.7)), то

$$
u_{\mu}(z)=\frac{(-1)^{r}}{(r-1) !} \int_{z}^{\tau_{+}}(t-z)^{r-1} u_{\mu}^{(r)}(t) d t, \quad z \in\left[\tau_{-}, \tau_{+}\right] .
$$

Применяя (3.28) для $s=r$, получаем

$$
\begin{aligned}
\left\|Q_{\mu}\right\|_{L_{q, v}\left(\widetilde{\Omega}_{\left[\tau_{-}, \tau_{+}\right]}\right)} & \underset{\mathfrak{Z}_{1}}{\lesssim}\left(\int_{\tau_{-}}^{\tau_{+}} v_{0}^{q}(z)\left|u_{\mu}(z)\right|^{q} \varphi(z)^{q|\mu|+d-1} d z\right)^{\frac{1}{q}} \\
& \leqslant C_{\tau_{-}, \tau_{+}}\left(\int_{\tau_{-}}^{\tau_{+}}\left|\frac{u_{\mu}^{(r)}(z)}{g_{0}(z)}\right|^{p} \varphi(z)^{p|\mu|+d-1} d z\right)^{\frac{1}{p}} \\
& \stackrel{(3.28)}{\lesssim} C_{\tau_{-}, \tau_{+}}\left(\int_{\tau_{-}}^{\tau_{+}} \int_{|y|<\varphi(z)}\left|\frac{\nabla^{r} u(y, z)}{g(y, z)}\right|^{p} d y d z\right)^{\frac{1}{p}} .
\end{aligned}
$$

Величина $C_{\tau_{-}, \tau_{+}}$оценивается с помощью теоремы В. Так как функция $\varphi$ неубывающая, получаем требуемую оценку (см. доказательство в $[6 ; \S 4]$ ). Таким образом,

$$
\|Q\|_{L_{q, v}\left(\widetilde{\Omega}_{\left[\tau_{-}, \tau_{+}\right]}\right)} \underset{\mathfrak{Z}_{1}}{\lesssim} A_{\left[\tau_{-}, \tau_{+}\right]}\left\|\frac{\nabla^{r} u}{g}\right\|_{L_{p}\left(\Omega_{\left[\tau_{-}, \tau_{+}\right]}\right)} .
$$

Оценим $\|u-Q\|_{L_{q, v}\left(\Omega_{\left[\tau_{-}, \tau_{+}\right]}\right)}$. Пусть

$$
\begin{gathered}
z_{0}=\tau_{+}, \quad z_{k+1}+\varphi\left(z_{k+1}\right)=z_{k}, \quad k \in \mathbb{Z}_{+}, \quad k_{*}=\min \left\{k \in \mathbb{Z}_{+}: z_{k+1}<\tau_{-}\right\}, \\
\widehat{z}_{k+1}=\max \left\{z_{k+1}, \tau_{-}\right\}, \quad \Omega_{(k)}=\bigcup_{z \in\left[\widehat{z}_{k+1}, z_{k}\right]} \Omega_{z}, \quad 0 \leqslant k \leqslant k_{*} .
\end{gathered}
$$

По лемме 2 существует $b=b\left(\mathfrak{Z}_{1}\right)>0$ такое, что $\Omega_{(k)} \in \mathbf{F C}(b)$. Пусть $g_{(k)}=$ $g_{0}\left(z_{k}\right), v_{(k)}=v_{0}\left(z_{k}\right)$. Из $(3.5)$ получаем

$$
g(x) \underset{\mathfrak{Z}_{1}}{\asymp} g_{(k)}, \quad v(x) \underset{\mathfrak{Z}_{1}}{\asymp} v_{(k)}
$$

для любого $x \in \Omega_{(k)}$. По построению

$$
z_{k}-z_{k+1}=\varphi\left(z_{k+1}\right) \underset{\mathfrak{Z}_{1}}{\asymp}\left(z_{k}\right)
$$

(здесь используется липшицевость $\varphi$ ). Отсюда, из теоремы С и неравенства (3.3) получаем

$$
\|u\|_{L_{q}\left(\Omega_{(k)}\right)} \underset{\widetilde{Z}_{1}}{\lesssim} \varphi\left(z_{k}\right)^{\frac{d}{q}-\frac{d}{p}}\left(\|u\|_{L_{p}\left(\Omega_{(k)}\right)}+\varphi\left(z_{k}\right)^{r}\left\|\nabla^{r} u\right\|_{L_{p}\left(\Omega_{(k)}\right)}\right) .
$$

Повторяя рассуждения в $[6 ; \S 4]$, получаем

$$
\|u-Q\|_{L_{q}\left(\Omega_{(k)}\right)} \underset{\mathfrak{Z}_{1}}{\lesssim} \varphi\left(z_{k}\right)^{r+\frac{d}{q}-\frac{d}{p}}\left\|\nabla^{r} u\right\|_{L_{p}\left(\Omega_{(k)}\right)} .
$$


Из (3.30) и леммы 6 следует, что

$$
\begin{aligned}
\|u-Q\|_{L_{q, v}\left(\Omega_{\left[\tau_{-}, \tau_{+}\right]}\right)} & \underset{\mathfrak{Z}_{1}}{\lesssim} \max _{0 \leqslant k \leqslant k_{*}} g_{(k)} v_{(k)} \varphi\left(z_{k}\right)^{r+\frac{d}{q}-\frac{d}{p}}\left\|\frac{\nabla^{r} u}{g}\right\|_{L_{p}\left(\Omega_{\left[\tau_{-}, \tau_{+}\right]}\right)} \\
& \stackrel{(3.26)}{\lesssim} A_{0,\left[\tau_{-}, \tau_{+}\right]}\left\|\frac{\nabla^{r} u}{g}\right\|_{L_{p}\left(\Omega_{\left[\tau_{-}, \tau_{+}\right]}\right)} \cdot
\end{aligned}
$$

Отсюда и из (3.29) получаем

$$
\|u\|_{L_{q, v}\left(\widetilde{\Omega}_{\left[\tau_{-}, \tau_{+}\right]}\right)} \underset{\mathfrak{Z}_{1}}{\lesssim} A_{\left[\tau_{-}, \tau_{+}\right]}\left\|\frac{\nabla^{r} u}{g}\right\|_{L_{p}\left(\Omega_{\left[\tau_{-}, \tau_{+}\right]}\right)} .
$$

Остается доказать, что

$$
\|u\|_{L_{q, v}\left(\Omega_{\left[\tau_{-}, \tau_{+}\right]} \backslash \widetilde{\Omega}_{\left[\tau_{-}, \tau_{+}\right]}\right)} \underset{\mathfrak{Z}_{1}}{\lesssim} A_{\left[\tau_{-}, \tau_{+}\right]}\left\|\frac{\nabla^{r} u}{g}\right\|_{L_{p}\left(\Omega_{\left[\tau_{-}, \tau_{+}\right]}\right)} .
$$

Положим

$$
\begin{aligned}
& \widetilde{\tau}_{-}=\sup \left\{t \in\left[\tau_{-}, \tau_{+}\right]: \exists(y, z) \in \Omega_{t}: z<\tau_{-}\right\} \\
& \widetilde{\tau}_{+}=\inf \left\{t \in\left[\tau_{-}, \tau_{+}\right]: \exists(y, z) \in \Omega_{t}: z>\tau_{+}\right\} .
\end{aligned}
$$

Тогда $\Omega_{\left[\tau_{-}, \tau_{+}\right]} \backslash \widetilde{\Omega}_{\left[\tau_{-}, \tau_{+}\right]} \subset \Omega_{\left[\tau_{-}, \widetilde{\tau}_{-}\right]} \cup \Omega_{\left[\widetilde{\tau}_{+}, \tau_{+}\right]}$. По лемме 3

$$
\varphi\left(\widetilde{\tau}_{-}\right) \underset{\mathfrak{Z}_{1}}{\asymp} \varphi\left(\tau_{-}\right), \quad \varphi\left(\widetilde{\tau}_{+}\right) \underset{\mathfrak{Z}_{1}}{\asymp} \varphi\left(\tau_{+}\right) .
$$

В силу (3.3) и (3.6)

$$
\widetilde{\tau}_{-}-\tau_{-} \underset{\mathfrak{Z}_{1}}{\asymp} \varphi\left(\widetilde{\tau}_{-}\right) \underset{\mathfrak{Z}_{1}}{\asymp} \varphi\left(\tau_{-}\right), \quad \tau_{+}-\widetilde{\tau}_{+} \underset{\mathfrak{Z}_{1}}{\asymp} \varphi\left(\widetilde{\tau}_{+}\right) \underset{\mathfrak{Z}_{1}}{\asymp} \varphi\left(\tau_{+}\right)
$$

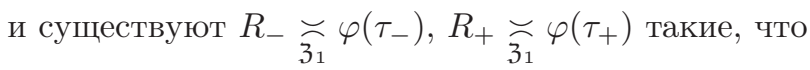

$$
B_{ \pm}:=B_{R_{ \pm}}\left(\eta_{\widetilde{\tau}_{ \pm}}\right) \subset \widetilde{\Omega}_{\left[\tau_{-}, \tau_{+}\right]} .
$$

По лемме 2

$$
\Omega_{\left[\tau_{-}, \widetilde{\tau}_{-}\right]} \in \mathbf{F C}\left(\widetilde{b}, \eta_{\widetilde{\tau}_{-}}\right), \quad \Omega_{\left[\widetilde{\tau}_{+}, \tau_{+}\right]} \in \mathbf{F C}\left(\widetilde{b}, \eta_{\widetilde{\tau}_{+}}\right),
$$

где $\widetilde{b}=\widetilde{b}\left(\mathfrak{Z}_{1}\right)>0$. Наконец, из леммы 5 следует, что

$$
\begin{aligned}
& g(x) \underset{\mathfrak{Z}_{1}}{\asymp} g_{0}\left(\tau_{-}\right), \quad v(x) \underset{\mathfrak{Z}_{1}}{\asymp} v_{0}\left(\tau_{-}\right) \quad \text { для любого } x \in \Omega_{\left[\tau_{-}, \widetilde{\tau}_{-}\right]}, \\
& g(x) \underset{\mathfrak{Z}_{1}}{\asymp} g_{0}\left(\tau_{+}\right), \quad v(x) \underset{\mathfrak{Z}_{1}}{\asymp} v_{0}\left(\tau_{+}\right) \quad \text { для любого } x \in \Omega_{\left[\widetilde{\tau}_{+}, \tau_{+}\right]} .
\end{aligned}
$$

По теореме $\mathrm{C}$

$$
\begin{gathered}
\|u\|_{L_{q, v}\left(\Omega_{\left[\tau_{-}, \tilde{\tau}_{-}\right]}\right)} \underset{\mathfrak{Z}_{1}}{\underset{\aleph_{\mathfrak{Z}_{1}}}{(3.26),(3.31),(3.32)}} A_{\left[\tau_{-}, \tau_{+}\right]}\left\|\frac{\nabla^{r} u}{g}\right\|_{L_{q, v}\left(B_{-}\right)}+g_{0}\left(\tau_{-}\right) v_{0}\left(\tau_{-}\right) \varphi\left(\tau_{-}\right)^{r+\frac{d}{q}-\frac{d}{p}}\left\|\frac{\nabla^{r} u}{g}\right\|_{L_{p}\left(\Omega_{\left[\tau_{-}, \tau_{+}\right]}\right)} \\
\end{gathered}
$$


и аналогично

$$
\|u\|_{L_{q, v}\left(\Omega_{\left[\tilde{\tau}_{+}, \tau_{+}\right]}\right)} \underset{\mathfrak{Z}_{1}}{\lesssim} A_{\left[\tau_{-}, \tau_{+}\right]}\left\|\frac{\nabla^{r} u}{g}\right\|_{L_{p}\left(\Omega_{\left[\tau_{-}, \tau_{+}\right]}\right)} .
$$

Теорема 2 доказана.

\section{СлЕДСТВИЕ 1. Пусть}

$$
\Omega \in \mathbf{F C}_{\varphi}(a), \quad 0<\lambda_{*}<1, \quad 0 \leqslant \tau_{-}<\tau_{+} \leqslant \frac{1}{2}, \quad \tau_{-}<\tau_{+}-\lambda_{*} \varphi\left(\tau_{+}\right) .
$$

Предположим, что $A_{\left[\tau_{-}, \tau_{+}\right]}<\infty$. Тогда $W_{p, g}^{r}\left(\Omega_{\left[\tau_{-}, \tau_{+}\right]}\right) \subset L_{q, v}\left(\Omega_{\left[\tau_{-}, \tau_{+}\right]}\right)$и существует линейный непрерывный оператор

$$
P: L_{q, v}\left(\Omega_{\left[\tau_{-}, \tau_{+}\right]}\right) \rightarrow \mathscr{P}_{r-1}\left(\Omega_{\left[\tau_{-}, \tau_{+}\right]}\right)
$$

такой, что для любой функции $f \in W_{p, g}^{r}\left(\Omega_{\left[\tau_{-}, \tau_{+}\right]}\right)$

$$
\|f-P f\|_{L_{q, v}\left(\Omega_{\left[\tau_{-}, \tau_{+}\right]}\right)} \underset{\mathcal{Z}_{1}, \lambda_{*}}{\lesssim} A_{\left[\tau_{-}, \tau_{+}\right]}\left\|\frac{\nabla^{r} f}{g}\right\|_{L_{p}\left(\Omega_{\left[\tau_{-}, \tau_{+}\right]}\right)} .
$$

ДокАзАТЕЛЬство. Пусть $\tau_{-} \geqslant \tau_{+}-\underline{R}_{\tau_{+}}$. Тогда

$$
\tau_{+}-\tau_{-} \stackrel{(3.1),(3.3)}{\leqslant} c_{0} \varphi\left(\tau_{+}\right) \underset{\mathfrak{Z}_{1}}{\lesssim} \varphi\left(\tau_{-}\right),
$$

где $c_{0}=c_{0}\left(\mathfrak{Z}_{1}\right)$. Проверим последнее неравенство. Так как $\varphi^{\prime}(t) \underset{t \rightarrow+0}{\rightarrow} 0$, то существует $\widehat{\tau}=\widehat{\tau}\left(\mathfrak{Z}_{1}\right)>0$ такое, что для $\tau_{+}<\widehat{\tau}$ выполнено

$$
\varphi\left(\tau_{-}\right) \geqslant \varphi\left(\tau_{+}-c_{0} \varphi\left(\tau_{+}\right)\right) \geqslant \frac{\varphi\left(\tau_{+}\right)}{2} .
$$

Если $\tau_{+}>\widehat{\tau}$, то $\underline{R}_{\tau_{+}} \leqslant \bar{R}_{\tau_{+}} \stackrel{(3.2)}{\leqslant} \kappa_{\Omega} \tau_{+}$и $\tau_{-} \geqslant\left(1-\kappa_{\Omega}\right) \tau_{+} \geqslant\left(1-\kappa_{\Omega}\right) \widehat{\tau}$. Значит,

$$
\varphi\left(\tau_{-}\right) \underset{\mathfrak{Z}_{1}}{\asymp} \underset{\mathfrak{Z}_{1}}{\asymp} \varphi\left(\tau_{+}\right) .
$$

Таким образом, $\tau_{+}-\tau_{-} \underset{\mathfrak{Z}_{1}}{\lesssim} \varphi\left(\tau_{-}\right)$. По лемме $2 \Omega_{\left[\tau_{-}, \tau_{+}\right]} \in \mathbf{F C}(\widetilde{b}), \widetilde{b} \underset{\mathfrak{Z}_{1}}{\asymp}$. Из неравенств $(3.2),(3.3)$ и леммы 5 следует, что

$$
g(x) \underset{\mathfrak{Z}_{1}}{\asymp} g_{0}\left(\tau_{+}\right), \quad v(x) \underset{\mathfrak{Z}_{1}}{\asymp} v_{0}\left(\tau_{+}\right)
$$

для любого $x \in \Omega_{\left[\tau_{-}, \tau_{+}\right]}$. Остается применить теорему С и неравенство (3.26).

Предположим, что $\tau_{-}<\tau_{+}-\underline{R}_{\tau_{+}}$. Пусть

$$
P_{0}: \operatorname{span} W_{p}^{r}\left(B_{\underline{R}_{\tau_{+}}}\left(\eta_{\tau_{+}}\right)\right) \rightarrow \mathscr{P}_{r-1}\left(B_{\underline{R}_{\tau_{+}}}\left(\eta_{\tau_{+}}\right)\right)
$$

- линейный непрерывный проектор. Тогда для любого $0 \leqslant k \leqslant r$ и любого $q_{k} \in[1,+\infty)$ такого, что $r-k+\frac{d}{q_{k}}-\frac{d}{p}>0$, выполнено

$$
\begin{gathered}
\left\|f-P_{0} f\right\|_{L_{q_{k}}\left(B_{\underline{R}_{\tau_{+}}}\left(\eta_{\tau_{+}}\right)\right)} \underset{p, q_{k}, r, d}{\lesssim} \underline{R}_{\tau_{+}}^{r-k+\frac{d}{q_{k}}-\frac{d}{p}}\left\|\nabla^{r} f\right\|_{L_{p}\left(B_{\underline{R}_{\tau_{+}}}\left(\eta_{\tau_{+}}\right)\right)} \\
\stackrel{(3.3)}{\underset{\mathfrak{Z}_{1}}{\delta}}\left(\varphi\left(\tau_{+}\right)\right)^{r-k+\frac{d}{q_{k}}-\frac{d}{p}}\left\|\nabla^{r} f\right\|_{L_{p}\left(B_{\underline{R}_{\tau_{+}}}\left(\eta_{\tau_{+}}\right)\right)}
\end{gathered}
$$

(см. [50]). 
Для того, чтобы определить $P_{0}$, берем ортогональную проекцию в пространстве $L_{2}\left(B_{\underline{R}_{\tau_{+}}}\left(\eta_{\tau_{+}}\right)\right)$на подпространство $\mathscr{P}_{r-1}\left(B_{\underline{R}_{\tau_{+}}}\left(\eta_{\tau_{+}}\right)\right)$, продолжаем ее на $L_{1}\left(B_{\underline{R}_{\tau_{+}}}\left(\eta_{\tau_{+}}\right)\right)$по непрерывности и затем ограничиваем на $L_{q, v}\left(B_{\underline{R}_{\tau_{+}}}\left(\eta_{\tau_{+}}\right)\right)$. Функция $P f$ определяется как продолжение полинома $P_{0}(f)$ на область $\Omega_{\left[\tau_{-}, \tau_{+}\right]}$. Тогда образ $P$ содержится в $\mathscr{P}_{r-1}\left(\Omega_{\left[\tau_{-}, \tau_{+}\right]}\right)$. Из условия $A_{\left[\tau_{-}, \tau_{+}\right]}<\infty$ следует, что $v \in L_{q}(\Omega)$ и оператор

$$
P: L_{q, v}\left(\Omega_{\left[\tau_{-}, \tau_{+}\right]}\right) \rightarrow L_{q, v}\left(\Omega_{\left[\tau_{-}, \tau_{+}\right]}\right)
$$

непрерывен.

Пусть $\psi_{0} \in C_{0}^{\infty}\left(\mathbb{R}^{d}\right), \operatorname{supp} \psi_{0} \subset B_{1}(0),\left.\psi_{0}\right|_{B_{1 / 2}(0)}=1, \psi_{0}(x) \in[0,1]$ для любого $x \in \mathbb{R}^{d}$. Положим

$$
R=\frac{\underline{R}_{\tau_{+}}}{2}, \quad \psi(x)=\psi_{0}\left(\frac{x-\eta_{\tau_{+}}}{\underline{R}_{\tau_{+}}}\right) .
$$

Имеем $\operatorname{supp} \psi \subset B_{\underline{R}_{\tau_{+}}}\left(\eta_{\tau_{+}}\right), \operatorname{supp}(1-\psi) \subset \Omega \backslash B_{R}\left(\eta_{\tau_{+}}\right)$. В силу условий $(3.2)$, (3.3), (3.5) и леммы 5

$$
g(x) \underset{\mathfrak{Z}_{1}}{\asymp} g_{0}\left(\tau_{+}\right), \quad v(x) \underset{\varlimsup_{1}}{\asymp} v_{0}\left(\tau_{+}\right), \quad x \in B_{\underline{R}_{\tau_{+}}}\left(\eta_{\tau_{+}}\right) .
$$

Значит,

$$
\begin{aligned}
& \|\psi(f-P f)\|_{L_{q, v}\left(\Omega_{\left[\tau_{-}, \tau_{+}\right]}\right)} \leqslant\|f-P f\|_{L_{q, v}\left(B_{\underline{R}_{\tau_{+}}}\left(\eta_{\tau_{+}}\right)\right)} \\
& \stackrel{\overbrace{\mathfrak{Z}_{1}}^{(3.33),}}{(3.34)} g_{0}\left(\tau_{+}\right) v_{0}\left(\tau_{+}\right)\left(\varphi\left(\tau_{+}\right)\right)^{\delta}\left\|\frac{\nabla^{r} f}{g}\right\|_{L_{p}\left(B_{\underline{R}_{\tau_{+}}}\left(\eta_{\tau_{+}}\right)\right)} \\
& \underset{\mathfrak{Z}_{1}}{\stackrel{(3.26)}{\lessgtr}} A_{\left[\tau_{-}, \tau_{+}\right]}\left\|\frac{\nabla^{r} f}{g}\right\|_{L_{p}\left(\Omega_{\left[\tau_{-}, \tau_{+}\right]}\right)} .
\end{aligned}
$$

Величина $\|(1-\psi)(f-P f)\|_{L_{q, v}\left(\Omega_{\left[\tau_{-}, \tau_{+}\right]}\right)}$оценивается по теореме 2 (рассуждения такие же, как в [52]). Следствие 1 доказано.

Пусть выполнены условия (1.3)-(1.8). Покажем, что

$$
A_{\left[\tau_{-}, \tau_{+}\right]} \leqslant A_{\left[0, \tau_{+}\right]} \underset{\mathfrak{Z}_{1}}{\lesssim}\left|\log \tau_{+}\right|^{-\alpha} \rho\left(\left|\log \tau_{+}\right|\right) .
$$

В самом деле, интегралы из определения $A_{i,\left[0, \tau_{+}\right]}, i=0,1$, легко оцениваются (для этого применяем лемму 1 и заменяем $z-t$ на $z$, a $t-z$ на $t$ ). Затем используем убывание функции $t^{-\alpha} \rho(t)$ при больших $t$.

Эту оценку будем использовать при $\tau_{-}<\frac{\tau_{+}}{2}$. Если $\tau_{-} \geqslant \frac{\tau_{+}}{2}$, то

$$
\varphi(t) \underset{\mathfrak{Z}_{1}}{\asymp} \varphi\left(\tau_{+}\right), \quad g_{0}(t) \underset{\mathfrak{Z}_{1}}{\asymp} g_{0}\left(\tau_{+}\right), \quad v_{0}(t) \underset{\mathfrak{Z}_{1}}{\asymp} v_{0}\left(\tau_{+}\right)
$$

для любого $t \in\left[\tau_{-}, \tau_{+}\right]$. Значит,

$$
\begin{aligned}
A_{\left[\tau_{-}, \tau_{+}\right]} \underset{\mathfrak{Z}_{1}}{\underset{1}{\lesssim}} g_{0}\left(\tau_{+}\right) v_{0}\left(\tau_{+}\right)\left[\varphi\left(\tau_{+}\right)\right]^{(d-1)\left(\frac{1}{q}-\frac{1}{p}\right)}\left(\tau_{+}-\tau_{-}\right)^{r+\frac{1}{q}-\frac{1}{p}} \\
=\left|\log \tau_{+}\right|^{-\alpha} \rho\left(\left|\log \tau_{+}\right|\right) \tau_{+}^{-r-\frac{1}{q}+\frac{1}{p}}\left(\tau_{+}-\tau_{-}\right)^{r+\frac{1}{q}-\frac{1}{p}} .
\end{aligned}
$$




\section{§4. Оценки поперечников}

Здесь мы предполагаем, что $p \leqslant q$.

Скажем, что множества $A, B \subset \mathbb{R}^{d}$ не перекрываются, если $A \cap B$ является множеством меры нуль.

Пусть $E, E_{1}, \ldots, E_{m} \subset \mathbb{R}^{d}$ - измеримые множества. Скажем, что $\left\{E_{i}\right\}_{i=1}^{m}$ является покрытием $E$, если множество $E \backslash\left(\bigcup_{i=1}^{m} E_{i}\right)$ имеет меру нуль. Назовем $\left\{E_{i}\right\}_{i=1}^{m}$ разбиением $E$, если множества $E_{i}$ попарно не перекрываются и множество $\left(\bigcup_{i=1}^{m} E_{i}\right) \triangle E$ имеет меру нуль.

Пусть $G \subset \Omega$ - измеримое множество, $T=\left\{G_{i}\right\}_{i=1}^{i_{0}}-$ конечное разбиение $G$. Обозначим

$$
\mathscr{S}_{r, T}(G)=\left\{S: \Omega \rightarrow \mathbb{R}:\left.S\right|_{G_{i}} \in \mathscr{P}_{r-1}\left(G_{i}\right), 1 \leqslant i \leqslant i_{0},\left.S\right|_{\Omega \backslash G}=0\right\} .
$$

Для каждой функции $f \in L_{q, v}(G)$ положим

$$
\|f\|_{p, q, T, v}=\left(\sum_{i=1}^{i_{0}}\|f\|_{L_{q, v}\left(G_{i}\right)}^{p}\right)^{\frac{1}{p}} .
$$

Через $L_{p, q, T, v}(G)$ обозначим пространство $L_{q, v}(G)$ с нормой $\|\cdot\|_{p, q, T, v}$. Заметим, что $\|f\|_{p, q, T, v} \geqslant\|f\|_{L_{q, v}(G)}$.

В работе [52] доказано следующее утверждение (точнее, более общий результат для весовых пространств). В невесовом случае О. В. Бесов [7] привел более простое доказательство.

Лемма 7. Пусть $а>0, G \subset \mathbb{R}^{d}, G \in \mathbf{F C}(a), n \in \mathbb{N}$. Тогда существует семейство разбиений $\left\{T_{m, n}(G)\right\}_{m \in \mathbb{Z}_{+}}$со следующими свойствами:

1) $\operatorname{card} T_{m, n}(G) \underset{a, d}{\lesssim} 2^{m} n$;

2) для любого $E \in T_{m, n}(G)$ существует линейный непрерывный оператор $P_{E}: L_{q}(E) \rightarrow \mathscr{P}_{r-1}(E)$ такой, что для любой функиии $f \in W_{p}^{r}(G)$

$$
\left\|f-P_{E} f\right\|_{L_{q}(E)} \underset{p, q, r, d, a}{\lesssim}\left(2^{m} n\right)^{-\frac{r}{d}-\frac{1}{q}+\frac{1}{p}}(\operatorname{mes} G)^{\frac{r}{d}+\frac{1}{q}-\frac{1}{p}}\left\|\nabla^{r} f\right\|_{L_{p}(E)} ;
$$

3) для любого $m \in \mathbb{Z}_{+}, E \in T_{m, n}(G)$

$$
\begin{aligned}
& \operatorname{card}\left\{E^{\prime} \in T_{m+1, n}(G): \operatorname{mes}\left(E \cap E^{\prime}\right)>0\right\} \underset{a, d}{\lesssim} 1, \\
& \operatorname{card}\left\{E^{\prime} \in T_{m-1, n}(G): \operatorname{mes}\left(E \cap E^{\prime}\right)>0\right\} \underset{a, d}{\lesssim} 1, \quad \text { ecлu } m \geqslant 1 .
\end{aligned}
$$

Пусть $X, Y$ - нормированные пространства, $B \subset X, A \in L(X, Y)$. Тогда

$$
d_{n}(A(B), Y) \leqslant\|A\| d_{n}(B, X) .
$$

Если $A-$ изоморфизм $X$ и $Y$, то

$$
\lambda_{n}(A(B), Y) \leqslant\|A\| \lambda_{n}(B, X), \quad d^{n}(A(B), Y) \leqslant\|A\| d^{n}(B, X) .
$$

Такие же неравенства выполнены, если $Y$ является подпространством в пространстве $X, B \subset Y$ и $A$ - линейный проектор на $Y$. Эти утверждения следуют из определений колмогоровского, гельфандовского и линейного поперечников.

Обозначим через $\chi_{E}$ характеристическую функцию множества $E$. 
Лемма 8. Пусть $\Omega \subset \mathbb{R}^{d}$ - область, $G_{1}, \ldots, G_{m} \subset \Omega$ - попарно не перекръвающиеся подобласти, $\psi_{1}, \ldots, \psi_{m} \in W_{p, g}^{r}(\Omega),\left\|\frac{\nabla^{r} \psi_{j}}{g}\right\|_{L_{p}(\Omega)}=1, \operatorname{supp} \psi_{j} \subset G_{j}$, $\left\|\psi_{j}\right\|_{L_{q, v}\left(G_{j}\right)} \geqslant M, 1 \leqslant j \leqslant m$. Тогда

$$
\vartheta_{n}\left(W_{p, g}^{r}(\Omega), L_{q, v}(\Omega)\right) \geqslant M \cdot \vartheta_{n}\left(B_{p}^{m}, l_{q}^{m}\right)
$$

(см. определение $\vartheta_{n}$ перед теоремой $\left.A\right)$.

ДокАЗАТЕльство. Пусть $X=\operatorname{span}\left\{\psi_{j}\right\}_{j=1}^{m} \subset L_{q, v}(\Omega)$. Из определения гельфандовского поперечника следует, что

$$
d^{n}\left(W_{p, g}^{r}(\Omega), L_{q, v}(\Omega)\right) \geqslant d^{n}\left(W_{p, g}^{r}(\Omega) \cap X, X\right) .
$$

Покажем, что

$$
\begin{aligned}
& d_{n}\left(W_{p, g}^{r}(\Omega), L_{q, v}(\Omega)\right) \geqslant d_{n}\left(W_{p, g}^{r}(\Omega) \cap X, X\right), \\
& \lambda_{n}\left(W_{p, g}^{r}(\Omega), L_{q, v}(\Omega)\right) \geqslant \lambda_{n}\left(W_{p, g}^{r}(\Omega) \cap X, X\right) .
\end{aligned}
$$

Для этого построим проектор $Q: L_{q, v}(\Omega) \rightarrow X$ такой, что $\|Q\| \leqslant 1$ и применим неравенства (4.1), (4.2). Пусть $X_{j}=\operatorname{span}\left\{\psi_{j}\right\}$. Обозначим через $L_{q, v}^{(j)}(G)$ множество функций из $L_{q, v}(G)$ с носителем в $G_{j}$. Так как $\operatorname{dim} X_{j}=1$, то существует проектор $Q_{j}: L_{q, v}^{(j)}(G) \rightarrow X_{j}$ такой, что $\left\|Q_{j}\right\| \leqslant 1$. Пусть $Q(f)=\sum_{j=1}^{m} Q_{j}\left(f \chi_{G_{j}}\right)$. Так как множества $G_{j}$ попарно не перекрываются, то $\|Q\| \leqslant 1$.

Определим изоморфизм $T: X \rightarrow \mathbb{R}^{m}$ формулой

$$
T\left(\sum_{j=1}^{m} c_{j} \psi_{j}\right)=\left(c_{1}, \ldots, c_{m}\right)
$$

Так как $\left\|\frac{\nabla^{r} \psi_{j}}{g}\right\|_{L_{p}(\Omega)}=1$, то включение $\sum_{j=1}^{m} c_{j} \psi_{j} \in W_{p, g}^{r}(\Omega)$ эквивалентно условию $\left(c_{1}, \ldots, c_{m}\right) \in B_{p}^{m}$. Значит, $T\left(W_{p, g}^{r}(\Omega) \cap X\right)=B_{p}^{m}$. Покажем, что

$$
\|T\|_{L_{q, v}(G) \rightarrow l_{q}^{m}} \leqslant M^{-1}
$$

В самом деле, если $f=\sum_{j=1}^{m} c_{j} \psi_{j}$, то $\|T f\|_{l_{q}^{m}}=\left(\sum_{j=1}^{m}\left|c_{j}\right|^{q}\right)^{\frac{1}{q}}$,

$$
\|f\|_{L_{q, v}(G)}=\left(\sum_{j=1}^{m}\left|c_{j}\right|^{q}\left\|\psi_{j}\right\|_{L_{q, v}\left(G_{j}\right)}^{q}\right)^{\frac{1}{q}} \geqslant M\|T f\|_{l_{q}^{m}} .
$$

В силу (4.1) и (4.2)

$$
\begin{aligned}
\vartheta_{n}\left(B_{p}^{m}, l_{q}^{m}\right) & =\vartheta_{n}\left(T\left(W_{p, g}^{r}(\Omega) \cap X\right), l_{q}^{m}\right) \\
& \leqslant\|T\|_{L_{q, v}(G) \rightarrow l_{q}^{m}} \vartheta_{n}\left(W_{p, g}^{r}(\Omega) \cap X, X\right) \leqslant M^{-1} \vartheta_{n}\left(W_{p, g}^{r}(\Omega) \cap X, X\right) .
\end{aligned}
$$

Отсюда следуют требуемые оценки.

ПредлОЖенИЕ 2. Пусть $G \subset \mathbb{R}^{d}$ - область. Предположим, ито $W_{p, g}^{r}(G) \subset$ $L_{q, v}(G)$. Пусть $M>0, m_{0} \in \mathbb{Z}_{+} \cup\{+\infty\} u\left\{T_{m}\right\}_{m=0}^{m_{0}}-$ семейство конечньх покрытий $G$ со следующими свойствами: 
(i) $\mathscr{N}_{T_{m}} \leqslant M$ для любого $m \in \overline{0, m_{0}}$;

(ii) для любых $m \in \overline{0, m_{0}}, E \in T_{m}$ существует линейный непрерывный оператор $P_{E, m}: L_{q, v}(E) \rightarrow \mathscr{P}_{r-1}(E)$ такой, что для любой функиии $f \in W_{p, g}^{r}(G)$ выполнено неравенство

$$
\left\|f-P_{E, m} f\right\|_{L_{q, v}(E)} \leqslant C_{m}\left\|\frac{\nabla^{r} f}{g}\right\|_{L_{p}(E)} ;
$$

(iii) $\operatorname{card}\left\{E^{\prime} \in T_{m \pm 1}: \operatorname{mes}\left(E \cap E^{\prime}\right)>0\right\} \leqslant M$ для любого $E \in T_{m}$.

Пусть $U \subset G$ - измеримое подмножество. Тогда существует последовательность разбиений $\left\{\widehat{T}_{m}\right\}_{m=0}^{m_{0}}$ множества $U$ такая, что

1) $\operatorname{card} \widehat{T}_{m} \leqslant \operatorname{card} T_{m}$;

2) существует линейный непрерывный оператор $P_{m}: L_{q, v}(G) \rightarrow \mathscr{S}_{r, \widehat{T}_{m}}(U)$ такой, что для любой функции $f \in W_{p, g}^{r}(G)$ выполнено неравенство

$$
\left\|f-P_{m} f\right\|_{p, q, \widehat{T}_{m}, v} \underset{M, p}{\lesssim} C_{m}\left\|\frac{\nabla^{r} f}{g}\right\|_{L_{p}(G)} ;
$$

3) $\operatorname{card}\left\{E^{\prime} \in \widehat{T}_{m \pm 1}: \operatorname{mes}\left(E \cap E^{\prime}\right)>0\right\} \leqslant M$ для любого $E \in \widehat{T}_{m}$;

4) существуют инбективные отображения $\mathscr{F}_{m}: \widehat{T}_{m} \rightarrow T_{m}$ такие, что для любого $E \in \widehat{T}_{m}$ выполнено включение $E \subset \mathscr{F}_{m}(E)$.

ДокАЗАТЕЛЬСтво. Пусть $T_{m}=\left\{E_{i, m}\right\}_{i=1}^{k_{m}}$. Обозначим

$$
\begin{gathered}
\widehat{E}_{1, m}=U \cap E_{1, m}, \quad \widehat{E}_{i, m}=U \cap E_{i, m} \backslash \bigcup_{j=1}^{i-1} E_{j, m}, \quad i \geqslant 2, \\
\widehat{T}_{m}=\left\{\widehat{E}_{i, m}\right\}_{i \in I_{m}}, \quad I_{m}=\left\{i \in \overline{1, k_{m}} \text { : mes } \widehat{E}_{i, m}>0\right\},
\end{gathered}
$$

$\mathscr{F}_{m}\left(\widehat{E}_{i, m}\right)=E_{i, m}, i \in I_{m}$. Тогда для $\widehat{T}_{m}$ выполнены свойства 1), 3) и 4). Определим оператор $P_{m}$ равенством $\left.P_{m} f\right|_{\widehat{E}_{i, m}}=\left.P_{E_{i, m}, m} f\right|_{\widehat{E}_{i, m}}, i \in I_{m}$. Используя условие $\mathscr{N}_{T_{m}} \leqslant M$, получаем свойство 2).

TеOрема 3. Пусть $\Omega \in \mathbf{F C}_{\varphi}(a), g(y, z)=g_{0}(z), v(y, z)=v_{0}(z), y \in \mathbb{R}^{d-1}$, $0<z \leqslant \frac{1}{2}$, и пусть выполнень условия (1.3)-(1.7)). Для $z \geqslant \frac{1}{2}$ продолжаем функиии $g_{0}, v_{0}$ и к константой. Тогда выполнены утверждения теоремы 1 для $\mathfrak{Z}=\left(\mathfrak{Z}_{1}, g, v\right)$.

ДоказАтельство. Оценка сверху. Достаточно рассмотреть случай $n=2^{N d}$, $N \in \mathbb{N}$. Обозначим

$$
D_{1}=\Omega_{\left[2^{-2}, 2^{-1}\right]}, \quad D_{j}=\Omega_{\left[2^{-j-1}, 2^{-j}\right]} \backslash \bigcup_{i=1}^{j-1} \Omega_{\left[2^{-i-1}, 2^{-i}\right]}, \quad j \geqslant 2 .
$$

Шаг 1. Рассмотрим $j \in \mathbb{N}, j \geqslant 2, l \in \mathbb{Z}_{+}$. Построим покрытие $\widetilde{\mathbf{R}}_{j, l}$ множества $\Omega_{\left[2^{-j}, 2^{-j+1}\right]}$. Без ограничения общности можно считать

$$
\varphi(z) \leqslant z \quad \text { для } \quad \forall z \in\left(0, \frac{1}{2}\right] .
$$


Пусть $l_{j} \in \mathbb{Z}_{+}$таковы, что

$$
2^{-j-l_{j}-1}<\varphi\left(2^{-j}\right) \leqslant 2^{-j-l_{j}} .
$$

Для $0 \leqslant l \leqslant l_{j}, 1 \leqslant i \leqslant 2^{l}$ положим

$$
\tau_{j, l}(i)=2^{-j}+i \cdot 2^{-j-l}, \quad \widetilde{\mathbf{R}}_{j, l}=\left\{U_{i, j, l}\right\}_{1 \leqslant i \leqslant 2^{l}}, \quad U_{i, j, l}=\Omega_{\left[\tau_{j, l}(i-1), \tau_{j, l}(i)\right]} .
$$

Тогда $\operatorname{card} \widetilde{\mathbf{R}}_{j, l}=2^{l}$. Далее, по лемме 6

$$
\mathscr{N}_{\widetilde{\mathbf{R}}_{j, l}} \underset{\mathfrak{Z}}{\lesssim} 1
$$

Отсюда и из определения $\widetilde{\mathbf{R}}_{j, l}$ получаем

$$
\operatorname{card}\left\{U^{\prime} \in \widetilde{\mathbf{R}}_{j, l \pm 1}: \operatorname{mes}\left(U^{\prime} \cap U\right)>0\right\} \underset{\mathfrak{Z}}{\lesssim} 1
$$

В силу следствия 1 и оценки (3.36) для любого $i \in \overline{1,2^{l}}$ существует линейный непрерывный оператор $P_{i}^{j, l}: L_{q, v}\left(U_{i, j, l}\right) \rightarrow \mathscr{P}_{r-1}\left(U_{i, j, l}\right)$ такой, что для любой функции $f \in W_{p, g}^{r}(\Omega)$

$$
\begin{aligned}
\left\|f-P_{i}^{j, l} f\right\|_{L_{q, v}\left(U_{i, j, l}\right)} & \underset{\mathfrak{Z}}{\lesssim} j^{-\alpha} \rho(j) \cdot 2^{-l\left(r+\frac{1}{q}-\frac{1}{p}\right)}\left\|\frac{\nabla^{r} f}{g}\right\|_{L_{p}\left(U_{i, j, l}\right)} \\
& \leqslant j^{-\alpha} \rho(j) \cdot 2^{-l\left(\frac{r}{d}+\frac{1}{q}-\frac{1}{p}\right)}\left\|\frac{\nabla^{r} f}{g}\right\|_{L_{p}\left(U_{i, j, l}\right)} .
\end{aligned}
$$

В силу предложения 2 для любого $l=\overline{0, l_{j}}$ существуют разбиение $\mathbf{R}_{j, l}$ множества $D_{j-1}$, инъективное отображение $\mathscr{F}_{j, l}: \mathbf{R}_{j, l} \rightarrow \widetilde{\mathbf{R}}_{j, l}$ и линейный непрерывный оператор $\mathbb{P}_{j, l}: L_{q, v}\left(\Omega_{\left[2^{-j}, 2^{-j+1}\right]}\right) \rightarrow \mathscr{S}_{r, \mathbf{R}_{j, l}}\left(D_{j-1}\right)$ такие, что

$$
\operatorname{card} \mathbf{R}_{j, l} \leqslant 2^{l},
$$

для любого $U \in \mathbf{R}_{j, l}$

$$
\operatorname{card}\left\{U^{\prime} \in \mathbf{R}_{j, l \pm 1}: \operatorname{mes}\left(U^{\prime} \cap U\right)>0\right\} \underset{\mathfrak{Z}}{\lesssim} 1
$$

и $U \subset \mathscr{F}_{j, l}(U)$; кроме того, для любой функции $f \in W_{p, g}^{r}\left(\Omega_{\left[2^{-j}, 2^{-j+1}\right]}\right)$

$$
\left\|f-\mathbb{P}_{j, l} f\right\|_{p, q, \mathbf{R}_{j, l}, v} \underset{\mathfrak{Z}}{\lesssim} j^{-\alpha} \rho(j) \cdot 2^{-l\left(\frac{r}{d}+\frac{1}{q}-\frac{1}{p}\right)}\left\|\frac{\nabla^{r} f}{g}\right\|_{L_{p}\left(\Omega_{\left[2^{-j}, 2^{-j+1}\right]}\right)} .
$$

В силу леммы 2 и неравенства (4.4) выполнено $U_{i, j, l_{j}} \in \mathbf{F C}\left(b_{*}\right)$ при $b_{*}=$ $b_{*}(\mathfrak{Z})>0$ и

$$
\operatorname{mes} U_{i, j, l_{j}} \underset{\mathfrak{Z}}{ } \varphi^{d}\left(2^{-j}\right) .
$$

Значит, по лемме 7 для любого $l \geqslant l_{j}$ существует разбиение $T_{l, j, i}=T_{l-l_{j}, 1}\left(U_{i, j, l_{j}}\right)$ такое, что

1) $\operatorname{card} T_{l, j, i} \underset{b_{*}, d}{\lesssim} 2^{l-l_{j}}$ 
2) для любого $E \in T_{l, j, i}$ существует линейный непрерывный оператор $P_{E}$ : $L_{q}(E) \rightarrow \mathscr{P}_{r-1}(E)$ такой, что для любой функции $f \in W_{p}^{r}(\Omega)$ выполнено

$$
\left\|f-P_{E} f\right\|_{L_{q}(E)} \underset{p, q, r, d, b_{*}}{\lesssim} 2^{-\left(l-l_{j}\right)\left(\frac{r}{d}+\frac{1}{q}-\frac{1}{p}\right)}\left(\operatorname{mes} U_{i, j, l_{j}}\right)^{\frac{r}{d}+\frac{1}{q}-\frac{1}{p}}\left\|\nabla^{r} f\right\|_{L_{p}(E)} ;
$$

3) для любого $l \geqslant l_{j}, G \in T_{l, j, i}$

$$
\begin{aligned}
& \operatorname{card}\left\{G^{\prime} \in T_{l+1, j, i}: \operatorname{mes}\left(G \cap G^{\prime}\right)>0\right\} \underset{b_{*}, d}{\lesssim} 1, \\
& \operatorname{card}\left\{G^{\prime} \in T_{l-1, j, i}: \operatorname{mes}\left(G \cap G^{\prime}\right)>0\right\} \underset{b_{*}, d}{\lesssim} 1, \quad \text { если } l \geqslant l_{j}+1 .
\end{aligned}
$$

Из (1.3)-(1.8), (4.4)-(4.10) и неравенства

$$
2^{\frac{\delta}{d} l_{j}} \leqslant 2^{\left(r+\frac{1}{q}-\frac{1}{p}\right) l_{j}}
$$

следует, что для любого $E \in T_{l, j, i}$

$$
\left\|f-P_{E} f\right\|_{L_{q, v}(E)} \underset{\mathfrak{Z}}{\lesssim} j^{-\alpha} \rho(j) \cdot 2^{-l\left(\frac{r}{d}+\frac{1}{q}-\frac{1}{p}\right)}\left\|\frac{\nabla^{r} f}{g}\right\|_{L_{p}(E)} .
$$

Пусть $D \in \mathbf{R}_{j, l_{j}}$. Обозначим через $i(D)$ число $i$ такое, что $U_{i, j, l_{j}}=\mathscr{F}_{j, l}(D)$. Для $l>l_{j}$ положим

$$
\mathbf{R}_{j, l}=\left\{D \cap E: D \in \mathbf{R}_{j, l_{j}}, E \in T_{l, j, i(D)}\right\} .
$$

Тогда $\mathbf{R}_{j, l}$ является разбиением $D_{j-1}$. В силу (4.6) и свойства 1$)$ разбиения $T_{l, j, i}$ имеем

$$
\operatorname{card} \mathbf{R}_{j, l} \underset{b_{*}, d}{\lesssim} 2^{l}
$$

Покажем, что для любого $U \in \mathbf{R}_{j, l}$

$$
\operatorname{card}\left\{U^{\prime} \in \mathbf{R}_{j, l \pm 1}: \operatorname{mes}\left(U^{\prime} \cap U\right)>0\right\} \underset{\mathfrak{Z}}{\lesssim} 1
$$

В самом деле, пусть $D, D^{\prime} \in \mathbf{R}_{j, l_{j}}, E \in T_{l, j, i(D)}, E^{\prime} \in T_{l \pm 1, j, i\left(D^{\prime}\right)}, \operatorname{mes}\left(D \cap E \cap D^{\prime} \cap\right.$ $\left.E^{\prime}\right)>0$. Так как $\mathbf{R}_{j, l_{j}}$ является разбиением, то $D^{\prime}=D$. Остается применить свойство 3) разбиения $T_{l, j, i}$.

Пусть $f \in L_{q, v}\left(\Omega_{\left[2^{-j}, 2^{-j+1}\right]}\right)$. Положим $\left.\mathbb{P}_{j, l} f\right|_{D \cap E}=\left.P_{E} f\right|_{D \cap E}$, где $D, E$ из (4.12) и $P_{E}$ из свойства 2) разбиения $T_{l, j, i(D)}$. Обозначим

$$
C_{j, l}=j^{-\alpha} \rho(j) \cdot 2^{-l\left(\frac{r}{d}+\frac{1}{q}-\frac{1}{p}\right)} .
$$

Тогда $\mathbb{P}_{j, l}: L_{q, v}\left(\Omega_{\left[2^{-j}, 2^{-j+1}\right]}\right) \rightarrow \mathscr{S}_{r, \mathbf{R}_{j, l}}\left(D_{j-1}\right)$ является линейным непрерывным оператором и для любой функции $f \in W_{p, g}^{r}\left(\Omega_{\left[2^{-j}, 2^{-j+1}\right]}\right)$

$$
\begin{aligned}
& \left\|f-\mathbb{P}_{j, l} f\right\|_{p, q, \mathbf{R}_{j, l}, v}=\left(\sum_{D \in \mathbf{R}_{j, l}} \sum_{E \in T_{l, j, i(D)}}\left\|f-P_{E} f\right\|_{L_{q, v}(D \cap E)}^{p}\right)^{\frac{1}{p}} \\
& \underset{\mathfrak{Z}}{\stackrel{(4.11)}{\lessgtr}} C_{j, l}\left(\sum_{D \in \mathbf{R}_{j, l_{j}}} \sum_{E \in T_{l, j, i(D)}}\left\|\frac{\nabla^{r} f}{g}\right\|_{L_{p}(E)}^{p}\right)^{\frac{1}{p}}=C_{j, l}\left(\sum_{D \in \mathbf{R}_{j, l_{j}}}\left\|\frac{\nabla^{r} f}{g}\right\|_{L_{p}\left(U_{i(D), j, l_{j}}\right)}^{p}\right)^{\frac{1}{p}} \\
& \leqslant C_{j, l}\left(\sum_{i=1}^{l_{j}}\left\|\frac{\nabla^{r} f}{g}\right\|_{L_{p}\left(U_{i, j, l_{j}}\right)}^{p}\right)^{\frac{1}{p}} \underset{\mathfrak{Z}}{\stackrel{(4.5)}{\lesssim}} C_{j, l}\left\|\frac{\nabla^{r} f}{g}\right\|_{L_{p}\left(\Omega_{\left[2^{-j}, 2^{-j+1}\right]}\right)}
\end{aligned}
$$


(предпоследнее неравенство следует из инъективности отображения $\mathscr{F}_{j, l_{j}}$ ). Таким образом,

$$
\left\|f-\mathbb{P}_{j, l} f\right\|_{p, q, \mathbf{R}_{j, l}, v} \underset{\mathfrak{Z}}{\lesssim} j^{-\alpha} \rho(j) \cdot 2^{-l\left(\frac{r}{d}+\frac{1}{q}-\frac{1}{p}\right)}\left\|\frac{\nabla^{r} f}{g}\right\|_{L_{p}\left(\Omega_{\left[2^{-j}, 2^{-j+1}\right]}\right)} .
$$

Шаг 2. Для $0 \leqslant t \leqslant N d$ положим $G_{t}:=\Omega_{\left[2^{-2^{t+1}}, 2^{-2^{t}}\right]}$,

$$
\widehat{G}_{0}=G_{0}, \quad \widehat{G}_{t}=G_{t} \backslash \bigcup_{s=0}^{t-1} G_{s}, \quad t \geqslant 1 .
$$

Тогда $\bigcup_{t=0}^{\infty} G_{t}=\bigcup_{t=0}^{\infty} \widehat{G}_{t}=\Omega$. Семейство областей $\left\{\Omega_{\left[2^{-j}, 2^{-j+1}\right]}\right\}_{2^{t}+1 \leqslant j \leqslant 2^{t+1}}$ образует покрытие $Q_{(t)}$ множества $G_{t}$. По лемме 6

$$
\mathscr{N}_{Q_{(t)}} \underset{\mathfrak{Z}}{\lesssim} 1 .
$$

Семейство $\left\{D_{j-1}\right\}_{2^{t}+1 \leqslant j \leqslant 2^{t+1}}$ образует покрытие множества $\widehat{G}_{t}$.

Пусть $t_{*}=t_{*}(N)=0$ или $t_{*}=t_{*}(N)=N d$ для каждого $N$, и пусть $\varepsilon>0$. Выбор $\varepsilon$ и $t_{*}$ (в зависимости от $\mathfrak{Z}$ ) будет сделан позже. Обозначим

$$
\begin{gathered}
m_{t}^{*}=\max \left\{\left\lceil t-N d+\varepsilon\left|t-t_{*}\right|\right\rceil, 0\right\}, \\
l_{m, t}=\left\lceil N d-t-\varepsilon\left|t-t_{*}\right|\right\rceil+m, \quad m \in \mathbb{Z}_{+}, \quad m \geqslant m_{t}^{*}, \\
\widehat{T}_{m, t, n}^{1}=\left\{U \in \mathbf{R}_{j, l_{m, t}}, 2^{t}+1 \leqslant j \leqslant 2^{t+1}\right\} .
\end{gathered}
$$

Тогда $\widehat{T}_{m, t, n}^{1}$ является разбиением множества $\widehat{G}_{t}$. В силу $(4.7)$ и (4.14) для любого $U \in \widehat{T}_{m, t, n}^{1}$

$$
\operatorname{card}\left\{U^{\prime} \in \widehat{T}_{m \pm 1, t, n}^{1}: \operatorname{mes}\left(U \cap U^{\prime}\right) \neq 0\right\} \underset{\mathfrak{Z}}{\lesssim} 1 .
$$

Из (4.6), (4.13) и (4.18) следует, что

$$
\operatorname{card} \widehat{T}_{m, t, n}^{1} \underset{\mathfrak{Z}}{\lesssim} 2^{t} \cdot 2^{l_{m, t}} \asymp n \cdot 2^{m-\varepsilon\left|t-t_{*}\right|} .
$$

Для $f \in L_{q, v}(\Omega)$ полагаем

$$
\left.P_{m, t, n}^{1} f\right|_{D_{j-1}}=\left.\mathbb{P}_{j, l_{m, t}} f\right|_{D_{j-1}}, \quad 2^{t}+1 \leqslant j \leqslant 2^{t+1},\left.\quad P_{m, t, n}^{1} f\right|_{\Omega \backslash \widehat{G}_{t}}=0 .
$$

Тогда $P_{m, t, n}^{1}: L_{q, v}(\Omega) \rightarrow \mathscr{S}_{r, \widehat{T}_{m, t, n}^{1}}\left(\widehat{G}_{t}\right)$ является линейным непрерывным оператором. Для любой функции $f \in W_{p, g}^{r}(\Omega)$ имеем

$$
\begin{aligned}
& \left\|f-P_{m, t, n}^{1} f\right\|_{p, q, \widehat{T}_{m, t, n}^{1}, v}=\left(\sum_{j=2^{t}+1}^{2^{t+1}}\left\|f-\mathbb{P}_{j, l_{m, t}} f\right\|_{p, q, \mathbf{R}_{j, l_{m, t}, v}}^{p}\right)^{\frac{1}{p}} \\
& \left.\underset{\mathfrak{Z}}{(4.8),(4.15)}\left(\sum_{j=2^{t}+1}^{2^{t+1}} j^{-p \alpha} \rho^{p}(j) \cdot 2^{-\frac{p \delta}{d} l_{m, t}}\left\|\frac{\nabla^{r} f}{g}\right\|_{L_{p}\left(\Omega_{[2-j, 2}-j+1\right]}\right)\right)^{\frac{1}{p}} \\
& \underset{\mathfrak{Z}}{\lesssim} 2^{-t \alpha} \rho\left(2^{t}\right) 2^{-\frac{\delta}{d} l_{m, t}}\left(\sum_{j=2^{t}+1}^{2^{t+1}}\left\|\frac{\nabla^{r} f}{g}\right\|_{L_{p}\left(\Omega_{\left[2^{-j}, 2^{-j+1}\right]}\right)}^{p}\right)^{\frac{1}{p}} \underset{\mathfrak{Z}}{\stackrel{(4.16)}{\lesssim}} 2^{-t \alpha} \rho\left(2^{t}\right) 2^{-\frac{\delta}{d} l_{m, t}} .
\end{aligned}
$$


Таким образом, для любой функции $f \in W_{p, g}^{r}(\Omega)$

$$
\left\|f-P_{m, t, n}^{1} f\right\|_{p, q, \widehat{T}_{m, t, n}^{1}, v} \underset{\mathfrak{Z}}{\lesssim} 2^{-t \alpha} \rho\left(2^{t}\right) 2^{-\frac{\delta}{d} l_{m, t}} .
$$

Шаг 3. Для $t \in \mathbb{Z}_{+}$положим $U_{t}=\Omega_{\left[0,2^{-2} 2^{t}\right]}$. В силу следствия 1 и неравенства (3.35) существует линейный непрерывный оператор $P^{t}: L_{q, v}\left(U_{t}\right) \rightarrow$ $\mathscr{P}_{r-1}\left(U_{t}\right)$ такой, что для любой функции $f \in W_{p, g}^{r}(\Omega)$

$$
\left\|f-P^{t} f\right\|_{L_{q, v}\left(U_{t}\right)} \underset{\mathfrak{Z}}{\lesssim} 2^{-t \alpha} \rho\left(2^{t}\right) .
$$

Шаг 4. Рассмотрим случаи $p=q$ и $p<q, \widehat{q} \leqslant 2$. Обозначим

$$
\widehat{U}_{t}=U_{t} \backslash \bigcup_{s=0}^{t-1} G_{s}, \quad t \in \mathbb{N}
$$

Положим $\left.P_{n}^{1} f\right|_{\widehat{G}_{t}}=\left.P_{m_{t}^{*}, t, n}^{1} f\right|_{\widehat{G}_{t}}$ для $0 \leqslant t \leqslant N d,\left.P_{n}^{1} f\right|_{\widehat{U}_{N d+1}}=\left.P^{N d+1} f\right|_{\widehat{U}_{N d+1}}$. Обозначим

$$
T_{n}^{1}=\left(\bigcup_{t=0}^{N d} \widehat{T}_{m_{t}^{*}, t, n}^{1}\right) \cup\left\{\widehat{U}_{N d+1}\right\} .
$$

Тогда $T_{n}^{1}$ является разбиением $\Omega$ и

$$
\operatorname{card} T_{n}^{1} \underset{\mathfrak{Z}}{\stackrel{(4.17),(4.20)}{\lesssim}} \sum_{0 \leqslant t \leqslant N d, m_{t}^{*}=0} n \cdot 2^{-\varepsilon\left|t-t_{*}\right|}+\sum_{0 \leqslant t \leqslant N d, m_{t}^{*}>0} 2^{t} \underset{\mathfrak{Z}, \varepsilon}{\lesssim} n .
$$

Для оценки колмогоровских и линейных поперечников достаточно получить оценку сверху для $\left\|f-P_{n}^{1} f\right\|_{L_{q, v}(\Omega)}$. Для оценки гельфандовских поперечников оценим сверху величину $\|f\|_{L_{q, v}(\Omega)}$ для $f \in W_{p, g}^{r}(\Omega)$ таких, что $P_{n}^{1} f=0$.

Имеем

$$
\begin{array}{r}
\left\|f-P_{n}^{1} f\right\|_{L_{q, v}(\Omega)}^{q}=\sum_{t=0}^{N d}\left\|f-P_{m_{t}^{*}, t, n}^{1} f\right\|_{L_{q, v}\left(\widehat{G}_{t}\right)}^{q}+\left\|f-P^{N d+1} f\right\|_{L_{q, v}\left(\widehat{U}_{N d+1}\right)}^{q} \\
\underset{\underbrace{\lessgtr}_{\mathfrak{Z}}}{(4.17),(4.22)} \sum_{t=0}^{N d} 2^{-t \alpha q} \rho^{q}\left(2^{t}\right) 2^{-\frac{\delta q}{d}\left(N d-t-\varepsilon\left|t-t_{*}\right|\right)}+n^{-\alpha q} \rho^{q}(n)=: S^{q}
\end{array}
$$

(здесь использовалось соотношение $\left.l_{m_{t}^{*}, t} \geqslant N d-t-\varepsilon\left|t-t_{*}\right|\right)$. Положим $\varepsilon=$ $\frac{d}{2 \delta}\left|\alpha-\frac{\delta}{d}\right|$. Если $\alpha>\frac{\delta}{d}$, то полагаем $t_{*}=0$ и получаем $S \underset{\mathfrak{Z}}{\lesssim} n^{-\frac{\delta}{d}} ;$ если $\alpha<\frac{\delta}{d}$, то берем $t_{*}=N d$ и получаем $S \underset{\mathfrak{Z}}{\lesssim} n^{-\alpha} \rho(n)$.

Шаг 5. Рассмотрим случай $p<q, \widehat{q}>2$. Обозначим $\widehat{t}_{N}=\left[\frac{\widehat{q}}{2} N d\right]$. Тогда при достаточно больших $N$ выполнено $N d+1<\widehat{t}_{N}$.

Для каждого $N d+1 \leqslant t<\widehat{t}_{N}, m \in \mathbb{Z}_{+}$, построим разбиение $\widehat{T}_{m, t, n}^{2}$ множества $\widehat{G}_{t}$.

Шаг 5.1. Пусть $\gamma=2^{1+\varepsilon}, m_{t}=\lceil(1+\varepsilon)(t-N d)\rceil, 0 \leqslant m \leqslant m_{t}$. Положим

$$
\begin{gathered}
j_{m, t}(s)=\left\lfloor\gamma^{t-N d} 2^{-m} s\right\rfloor, \quad \tau_{m, t}(s)=2^{-j_{m, t}(s)}, \quad s \in \mathbb{Z}_{+}, \\
J_{m, t}=\left\{s \in \mathbb{Z}_{+}: 2^{t} \leqslant j_{m, t}(s) \leqslant 2^{t+1}-1\right\} .
\end{gathered}
$$


Тогда card $J_{m, t} \underset{\mathfrak{Z}}{\lesssim} 2^{m} n \cdot 2^{-\varepsilon(t-N d)}$ для достаточно малых $\varepsilon$.

Обозначим через $T_{m, t, n}^{2}$ покрытие $G_{t}$ множествами $\Omega_{\left[\tau_{m, t}(s+1), \tau_{m, t}(s)\right]}, s \in$ $J_{m, t}, \tau_{m, t}(s) \neq \tau_{m, t}(s+1)$. Тогда

$$
\operatorname{card} T_{m, t, n}^{2} \underset{\mathfrak{Z}}{\lesssim} 2^{m} n \cdot 2^{-\varepsilon(t-N d)} .
$$

Если $\tau_{m, t}(s) \neq \tau_{m, t}(s+1)$, то

$$
\tau_{m, t}(s)-\tau_{m, t}(s+1) \asymp \tau_{m, t}(s) \stackrel{(4.3)}{\geqslant} \varphi\left(\tau_{m, t}(s)\right) .
$$

Поэтому из определения $\tau_{m, t}(s)$ и из леммы 6 следует, что для любого множества $U \in T_{m, t, n}^{2}$

$$
\operatorname{card}\left\{U^{\prime} \in T_{m+l, t, n}^{2}: \operatorname{mes}\left(U \cap U^{\prime}\right)>0\right\} \underset{\mathfrak{Z}}{\lesssim} 1, \quad l=0,1,-1 .
$$

В силу следствия 1 и неравенства (3.35) для любого множества $E \in T_{m, t, n}^{2}$ существует линейный непрерывный оператор $P_{E}: L_{q, v}(E) \rightarrow \mathscr{P}_{r-1}(E)$ такой, что для любой функции $f \in W_{p, g}^{r}(\Omega)$

$$
\left\|f-P_{E} f\right\|_{L_{q, v}(E)} \underset{\mathfrak{Z}}{\lesssim} 2^{-t \alpha} \rho\left(2^{t}\right)\left\|\frac{\nabla^{r} f}{g}\right\|_{L_{p}(E)} .
$$

В силу предложения 2 существуют разбиение $\widehat{T}_{m, t, n}^{2}$ множества $\widehat{G}_{t}$ и инъекция $\mathscr{F}_{m, t, n}: \widehat{T}_{m, t, n}^{2} \rightarrow T_{m, t, n}^{2}$ такие, что

$$
\operatorname{card} \widehat{T}_{m, t, n}^{2} \underset{\mathfrak{Z}}{\lesssim} 2^{m} n \cdot 2^{-\varepsilon(t-N d)}
$$

и для любого $U \in \widehat{T}_{m, t, n}^{2}$

$$
\operatorname{card}\left\{U^{\prime} \in \widehat{T}_{m \pm 1, t, n}^{2}: \operatorname{mes}\left(U \cap U^{\prime}\right)>0\right\} \underset{\mathfrak{Z}}{\lesssim} 1,
$$

$U \subset \mathscr{F}_{m, t, n}(U)$. Кроме того, существует линейный непрерывный оператор $P_{m, t, n}^{2}: L_{q, v}(\Omega) \rightarrow \mathscr{S}_{r, \widehat{T}_{m, t, n}^{2}}\left(\widehat{G}_{t}\right)$ такой, что для любой функции $f \in W_{p, g}^{r}(\Omega)$

$$
\left\|f-P_{m, t, n}^{2} f\right\|_{p, q, \widehat{T}_{m, t, n}^{2}, v} \underset{\mathfrak{Z}}{\lesssim} 2^{-t \alpha} \rho\left(2^{t}\right) .
$$

Заметим, что $\widehat{T}_{m, t, n}^{2}$ может быть определено следующим образом:

$$
\begin{gathered}
E \in \widehat{T}_{m, t, n}^{2} \Longleftrightarrow \operatorname{mes} E>0 \quad \text { и } \quad \exists s \in J_{m, t}: \tau_{m, t}(s) \neq \tau_{m, t}(s+1), \\
E=\widehat{G}_{t} \cap \Omega_{\left[\tau_{m, t}(s+1), \tau_{m, t}(s)\right]} \bigcup_{s^{\prime} \in J_{m, t}, s^{\prime}<s} \Omega_{\left[\tau_{m, t}\left(s^{\prime}+1\right), \tau_{m, t}\left(s^{\prime}\right)\right]} ; \\
\text { тогда } \mathscr{F}_{m, t, n}(E)=\Omega_{\left[\tau_{m, t}(s+1), \tau_{m, t}(s)\right]}
\end{gathered}
$$

(см. доказательство предложения 2). 
Шаг 5.2. Построим разбиение $\widehat{T}_{m, t, n}^{2}$ для $m>m_{t}$. Заметим, что для любого $s \in J_{m_{t}, t}$ выполнено неравенство $\left|j_{m_{t}, t}(s+1)-j_{m_{t}, t}(s)\right| \leqslant 1$. Поэтому каждый элемент покрытия $T_{m_{t}, t, n}^{2}$ совпадает с $\Omega_{\left[2^{-j}, 2^{-j+1}\right]}$ для некоторого $j \in\left\{2^{t}+\right.$ $\left.1, \ldots, 2^{t+1}\right\}$ (см. (4.23)). Напомним, что $\widehat{G}_{t} \subset G_{t}=\Omega_{\left[2^{-2^{t+1}, 2^{-2}}\right.}$. Отсюда и из (4.27) получаем

$$
\widehat{T}_{m_{t}, t, n}^{2}=\left\{D_{j-1}: 2^{t}+1 \leqslant j \leqslant 2^{t+1}, \quad \operatorname{mes} D_{j-1}>0\right\}
$$

и $\mathscr{F}_{m_{t}, t, n}\left(D_{j-1}\right)=\Omega_{\left[2^{-j}, 2^{-j+1}\right]}$.

Положим

$$
\widehat{T}_{m, t, n}^{2}=\left\{E \in \mathbf{R}_{j, m-m_{t}}: 2^{t}+1 \leqslant j \leqslant 2^{t+1}\right\} .
$$

Тогда $\widehat{T}_{m, t, n}^{2}$ является разбиением $\widehat{G}_{t}$. Из (4.6), (4.13) и (4.28) следует, что

$$
\operatorname{card} \widehat{T}_{m, t, n}^{2} \lesssim 2^{m-m_{t}} \cdot 2^{t} \asymp 2^{m} n \cdot 2^{-\varepsilon(t-N d)} .
$$

В силу (4.7) и (4.14) для любого $E \in \widehat{T}_{m, t, n}^{2}$,

$$
\operatorname{card}\left\{E^{\prime} \in \widehat{T}_{m \pm 1, t, n}^{2}: \operatorname{mes}\left(E \cap E^{\prime}\right)>0\right\} \underset{\mathfrak{Z}}{\lesssim} 1 .
$$

Для любой функции $f \in L_{q, v}(\Omega)$ положим $\left.P_{m, t, n}^{2} f\right|_{D_{j-1}}=\left.\mathbb{P}_{j, m-m_{t}} f\right|_{D_{j-1}}, 2^{t}+$ $1 \leqslant j \leqslant 2^{t+1},\left.P_{m, t, n}^{2} f\right|_{\Omega \backslash \widehat{G}_{t}}=0$. Тогда $P_{m, t, n}^{2}: L_{q, v}(\Omega) \rightarrow \mathscr{S}_{r, \widehat{T}_{m, t, n}^{2}}\left(\widehat{G}_{t}\right)$ является линейным непрерывным оператором и для любой функции $f \in W_{p, g}^{r}(\Omega)$

$$
\begin{aligned}
\left\|f-P_{m, t, n}^{2} f\right\|_{p, q, \widehat{T}_{m, t, n}^{2}, v} & =\left(\sum_{j=2^{t}+1}^{2^{t+1}}\left\|f-\mathbb{P}_{j, m-m_{t}} f\right\|_{p, q, \mathbf{R}_{j, m-m_{t}}, v}^{p}\right)^{\frac{1}{p}} \\
& \sum_{\mathfrak{Z}}^{(4.8),(4.15)}\left(\sum_{j=2^{t}+1}^{2^{t+1}} j^{-p \alpha} \rho^{p}(j) \cdot 2^{-\frac{p \delta}{d}\left(m-m_{t}\right)}\left\|\frac{\nabla^{r} f}{g}\right\|_{L_{p}\left(\Omega_{\left[2^{-j}, 2^{-j+1_{j}}\right.}\right)}^{p}\right)^{\frac{1}{p}} \\
& \underset{\mathfrak{Z}}{\lesssim} 2^{-t \alpha-\left(m-m_{t}\right) \frac{\delta}{d}} \rho\left(2^{t}\right)\left(\sum_{j=2^{t}+1}^{\frac{1}{p}}\left\|\frac{\nabla^{r} f}{g}\right\|_{L_{p}\left(\Omega_{\left[2^{-j}, 2^{-j+1}\right]}\right)}^{p}\right. \\
& \underset{(4.16)}{\lesssim} 2^{-t \alpha-\left(m-m_{t}\right) \frac{\delta}{d}} \rho\left(2^{t}\right) .
\end{aligned}
$$

Таким образом, для любой функции $f \in W_{p, g}^{r}(\Omega)$

$$
\left\|f-P_{m, t, n}^{2} f\right\|_{p, q, \widehat{T}_{m, t, n}^{2}, v} \underset{\mathfrak{Z}}{\lesssim} 2^{-t \alpha-\left(m-m_{t}\right) \frac{\delta}{d}} \rho\left(2^{t}\right) .
$$

Шаг 5.3. Пусть $f \in W_{p, g}^{r}(\Omega)$. Тогда

$$
\left\|f-P^{\widehat{t}_{N}} f\right\|_{L_{q, v}\left(U_{\widehat{t}_{n}}\right)} \underset{\mathfrak{Z}}{\stackrel{(4.22)}{\lesssim}} n^{-\frac{\alpha \widehat{q}}{2}} \rho\left(n^{\frac{\widehat{q}}{2}}\right) .
$$

Дальнейшие рассуждения такие же, как в работе [42]; здесь используются (4.19)-(4.21), (4.24)-(4.26), (4.29)-(4.32). 
Оценка снизу. Заметим, что $\Omega$ содержит куб $Q$ со стороной длины $\lambda \underset{\mathfrak{Z}}{\gtrsim}$, такой что $g(x) \underset{\mathfrak{Z}}{\gtrsim} 1, v(x) \underset{\mathfrak{Z}}{\gtrsim} 1$ для любого $x \in Q$. Значит,

$$
\vartheta_{n}\left(W_{p, g}^{r}(\Omega), L_{q, v}(\Omega)\right) \underset{\mathfrak{Z}}{\gtrsim} \vartheta_{n}\left(W_{p}^{r}\left([0,1]^{d}\right), L_{q}\left([0,1]^{d}\right)\right) .
$$

Применяя теорему А, получаем

$$
\begin{gathered}
\vartheta_{n}\left(W_{p, g}^{r}(\Omega), L_{q, v}(\Omega)\right) \underset{\mathfrak{Z}}{\gtrsim} n^{-\frac{\delta}{d}} \quad \text { при } p=q \text { или } p<q, \quad \widehat{q} \leqslant 2, \\
\vartheta_{n}\left(W_{p, g}^{r}(\Omega), L_{q, v}(\Omega)\right) \underset{\widetilde{\mathfrak{Z}}}{\gtrsim} n^{-\min \left\{\theta_{1}, \theta_{2}\right\}} \quad \text { при } p<q, \quad \widehat{q}>2 .
\end{gathered}
$$

Пусть $\psi \in C_{0}^{\infty}(0,1), \psi \geqslant 0, \int_{0}^{1}\left|\psi^{(r)}\right|^{p} d x=1$. Для $j \in \mathbb{N}$ положим $\psi_{j}\left(x^{\prime}, x_{d}\right)=$ $c_{j} \psi\left(2^{j} x_{d}-1\right)$, где $c_{j}>0$ выбирается так, что

$$
\left\|\frac{\nabla^{r} \psi_{j}}{g}\right\|_{L_{p}(\Omega)}=1 .
$$

Тогда

$$
\operatorname{supp} \psi_{j} \subset\left\{\left(x^{\prime}, x_{d}\right) \in \Omega: 2^{-j}<x_{d}<2^{-j+1}\right\} .
$$

Из (3.14) и порядковых равенств

$$
g(x) \stackrel{(1.3)}{\widehat{\mathfrak{Z}}} g\left(2^{-j}\right), \quad v(x) \stackrel{(1.3)}{\overleftarrow{\mathfrak{Z}}} v\left(2^{-j}\right), \quad 2^{-j}<x_{d}<2^{-j+1}
$$

следует, что

$$
\begin{gathered}
c_{j} \underset{\mathfrak{Z}, \psi}{\asymp} g\left(2^{-j}\right) \varphi\left(2^{-j}\right)^{-\frac{d-1}{p}} 2^{\frac{j}{p}} \cdot 2^{-j r}, \\
\left\|\psi_{j}\right\|_{L_{q, v}(\Omega)}^{\underset{\mathfrak{Z}, \psi}{\asymp}} c_{j} \cdot v\left(2^{-j}\right) \varphi\left(2^{-j}\right)^{\frac{d-1}{q}} 2^{-\frac{j}{q}} \asymp j^{-\alpha} \rho(j)
\end{gathered}
$$

(последнее соотношение следует из (1.3)-(1.8)). Если $2^{t} \leqslant j<2^{t+1}$, то

$$
\left\|\psi_{j}\right\|_{L_{q, v}(\Omega)} \asymp 2^{-t \alpha} \rho\left(2^{t}\right) .
$$

Применяя лемму 8, получаем

$$
\vartheta_{n}\left(W_{p, g}^{r}(\Omega), L_{q, v}(\Omega)\right) \underset{\mathfrak{Z}}{\gtrsim} 2^{-t \alpha} \rho\left(2^{t}\right) \vartheta_{n}\left(B_{p}^{2^{t}}, l_{q}^{2^{t}}\right) .
$$

Пусть $t=N d+1$. Тогда в силу (2.1)-(2.4)

$$
\begin{aligned}
\vartheta_{n}\left(W_{p, g}^{r}(\Omega), L_{q, v}(\Omega)\right) & \underset{\widetilde{\mathfrak{Z}}}{\gtrsim} n^{-\alpha} \rho(n) d_{n}\left(B_{p}^{2 n}, l_{q}^{2 n}\right) \\
& \underset{p, q}{\asymp} \begin{cases}n^{-\alpha} \rho(n), & p=q \text { или } p<q, \widehat{q} \leqslant 2, \\
n^{-\alpha-\min \left\{\frac{1}{p}-\frac{1}{q}, \frac{1}{2}-\frac{1}{\hat{q}}\right\}} \rho(n), & \widehat{q}>2 .\end{cases}
\end{aligned}
$$


Пусть $t=\left[\frac{\widehat{q}}{2} N d\right], \widehat{q}>2$. Тогда

$$
\vartheta_{n}\left(W_{p, g}^{r}(\Omega), L_{q, v}(\Omega)\right) \underset{\mathfrak{Z}}{\gtrsim} n^{-\frac{\widehat{q} \alpha}{2}} \rho\left(n^{\frac{\widehat{q}}{2}}\right) \vartheta_{n}\left(B_{p}^{\left[n^{\frac{\widehat{q}}{2}}\right]}, l_{q}^{\left[n^{\frac{\widehat{q}}{2}}\right]}\right) \underset{\mathfrak{Z}}{\asymp} n^{-\frac{\widehat{q} \alpha}{2}} \rho\left(n^{\frac{\widehat{q}}{2}}\right) .
$$

Теорема 3 доказана.

Из теоремы 3 и предложения 1 следует теорема 1.

В заключение автор выражает благодарность А. С. Кочурову и В. Ю. Протасову за ценные замечания при просмотре рукописи.

\section{Список литературы}

[1] О.В. Бесов, "Интегральные представления функций и теоремы вложения для области с условием гибкого рога", Тр. МИАН СССР, 170 (1984), 12-30; англ. пер.: O. V. Besov, "Integral representations of functions, and imbedding theorems for a region with flexible horn condition", Proc. Steklov Inst. Math., 170 (1987), 11-31.

[2] Д. А. Лабутин, "Интегральное представление функций и вложение пространств Соболева на областях с нулевыми углами", Матем. заметки, 61:2 (1997), 201-219; англ. пер.: D. A. Labutin, "Integral representations of functions and embeddings of Sobolev spaces on cuspidal domains", Math. Notes, 61:2 (1997), 164-179.

[3] Д. А. Лабутин, "Вложение пространств Соболева на гёльдеровых областях", Исследования по теории дифферениируемых функиий многих переменных и ее приложениям. Часть 18, Тр. МИАН, 227, Наука, М., 1999, 170-179; англ. пер.: D. A. Labutin, "Embedding of Sobolev spaces on Hölder domains", Proc. Steklov Inst. Math., 227 (1999), 163-172.

[4] P. Hajłasz, P. Koskela, "Isoperimetric inequalities and imbedding theorems in irregular domains", J. London Math. Soc. (2), 58:2 (1998), 425-450.

[5] T. Kilpeläinen, J. Malý, "Sobolev inequalities on sets with irregular boundaries", Z. Anal. Anwendungen, 19:2 (2000), 369-380.

[6] В. Г. Мазья, С. В. Поборчий, Алгебра и анализ, 18:4 (2006), 95-126; англ. пер.: V. G. Maz'ya, S. V. Poborchi, "Imbedding theorems for Sobolev spaces on domains with peak and on Hölder domains", St. Petersburg Math. J., 18:4 (2007), 583-605.

[7] О.В.Бесов, "О колмогоровских поперечниках классов Соболева на нерегулярной области", Ортогональные ряды, теория приближений и смежные вопросы, Сборник статей. K 60-летию со дня рождения академика Бориса Сергеевича Кашина, Тр. МИАН, 280, МАИК, М., 2013, 41-52; англ. пер.: О. V. Besov, "Kolmogorov widths of Sobolev classes on an irregular domain", Proc. Steklov Inst. Math., 280 (2013), 34-45.

[8] Х. Трибель, Теория интерполяиии, функииональные пространства, дифференциальные операторы, Мир, М., 1980, 660 с.; пер. с англ.: H. Triebel, Interpolation theory, function spaces, differential operators, VEB Deutscher Verlag der Wissenschaften, Berlin, 1978, 528 pp.

[9] A. Kufner, Weighted Sobolev spaces, Teubner-Texte Math., 31, BSB B. G. Teubner Verlagsgesellschaft, Leipzig, 1980, $151 \mathrm{pp.}$

[10] D. E. Edmunds, H. Triebel, Function spaces, entropy numbers, differential operators, Cambridge Tracts in Math., 120, Cambridge Univ. Press, Cambridge, 1996, xii +252 pp.

[11] H. Triebel, Theory of function spaces, v. III, Monogr. Math., 100, Birkhäuser Verlag, Basel, 2006, xii+426 pp. 
[12] D. E. Edmunds, W.D. Evans, Hardy operators, function spaces and embeddings, Springer Monogr. Math., Springer-Verlag, Berlin, 2004, xii+326 pp.

[13] Л.Д.Кудрявцев, С.М.Никольский, "Пространства дифференцируемых функций многих переменных и теоремы вложения", Анализ - 3, Итоги науки и техн. Сер. Соврем. пробл. мат. Фундам. направления, 26, ВИНИТИ, М., 1988, 5-157; англ. пер.: L. D. Kudryavtsev, S. M. Nikol'skij, "Spaces of differentiable functions of several variables and imbedding theorems", Analysis III. Spaces of differentiable functions, Encyclopaedia Math. Sci., 26, 1991, 1-140.

[14] В.Г. Мазья, "Классы областей и теоремы вложения функциональных пространств", Докл. АН ССCP, 133:3 (1960), 527-530; англ. пер.: V. G. Maz’ja, "Classes of domains and imbedding theorems for function spaces", Soviet Math. Dokl., 1 (1960), 882-885.

[15] Ю. Г. Решетняк, "Интегральные представления дифференцируемых функций в областях с негладкой границей”, Сиб. матем. журнал, 21:6 (1980), 108-116; англ. пер.: Yu. G. Reshetnyak, "Integral representations of differentiable functions in domains with nonsmooth boundary", Siberian Math. J., 21:6 (1981), 833-839.

[16] Ю.Г. Решетняк, "Замечание об интегральных представлениях дифференцируемых функций многих переменных", Сиб. матем. журнал, 25:5 (1984), 198-200.

[17] B. Bojarski, "Remarks on Sobolev imbedding inequalities", Complex analysis (Joensuu, 1987), Lecture Notes in Math., 1351, Springer, Berlin, 1988, 52-68.

[18] О.В. Бесов, "Вложение пространств Соболева на областях с условием распадающегося гибкого конуса", Исследования по теории дифферениируемых функиий многих переменных и ее приложениям. Часть 11, Сборник работ, Тр. МИАН CCCP, 173, 1986, 14-31; англ. пер.: O. V. Besov, "Imbedding of Sobolev spaces on domains with the splitting flexible cone condition", Proc. Steklov Inst. Math., 173 (1987), 13-30.

[19] W. D. Evans, D. J. Harris, "Fractals, trees and the Neumann Laplacian", Math. Ann., 296:3 (1993), 493-527.

[20] О.В. Бесов, "О компактности вложений весовых пространств Соболева на области с нерегулярной границей”, Функииональные пространства, гармонический анализ, дифференциалъные уравнения, Сборник статей. К 95-летию со дня рождения академика Сергея Михайловича Никольского, Тр. МИАН, 232, Наука, М., 2001, 72-93; англ. пер.: O. V. Besov, "On the compactness of embeddings of weighted Sobolev spaces on a domain with irregular boundary", Proc. Steklov Inst. Math., 232 (2001), 66-87.

[21] О. В. Бесов, “Теорема вложения Соболева для области с нерегулярной границей”, Матем. сб., 192:3 (2001), 3-26; англ. пер.: O. V. Besov, "Sobolev's embedding theorem for a domain with irregular boundary", Sb. Math., 192:3 (2001), 323-346.

[22] О.В. Бесов, "О компактности вложений весовых пространств Соболева на области с нерегулярной границей”, Докл. РАН, 376:6 (2001), 727-732; англ. пер.: O.V. Besov, "On the compactness of embeddings of weighted Sobolev spaces on a domain with an irregular boundary", Dokl. Math., 63:1 (2001), 95-100.

[23] О.В. Бесов, "Интегральные оценки дифференцируемых функций на нерегулярных областях", Матем. сб., 201:12 (2010), 69-82; англ. пер.: O. V. Besov, "Integral estimates for differentiable functions on irregular domains", Sb. Math., 201:12 (2010), 1777-1790.

[24] O. V. Besov, "Sobolev's embedding theorem for anisotropically irregular domains", Eurasian Math. J., 2:1 (2011), 32-51.

[25] L. Caso, R. D'Ambrosio, "Weighted spaces and weighted norm inequalities on irregular domains", J. Approx. Theory, 167 (2013), 42-58.

[26] A. Pietsch, "s-numbers of operators in Banach spaces", Studia Math., 51 (1974), 201-223. 
[27] S. Heinrich, "On the relation between linear $n$-widths and approximation numbers", J. Approx. Theory, 58:3 (1989), 315-333.

[28] D. E. Edmunds, J. Lang, "Gelfand numbers and widths", J. Approx. Theory, 166 (2013), 78-84.

[29] В. М. Тихомиров, Некоторые вопросы теории приближений, Изд-во МГУ, М., 1976, $304 \mathrm{c}$.

[30] В. М. Тихомиров, “Теория приближений”, Анализ - 2, Итоги науки и техн. Сер. Соврем. пробл. мат. Фундам. направления, 14, ВИНИТИ, М., 1987, 103-260.

[31] A. Pinkus, n-widths in approximation theory, Ergeb. Math. Grenzgeb. (3), 7, Springer-Verlag, Berlin, 1985, x+291 pp.

[32] В. М. Тихомиров, "Поперечники множеств в функциональных пространствах и теория наилучших приближений”, УМH, 15:3(93) (1960), 81-120; англ. пер.: V.M. Tikhomirov, "Diameters of sets in function spaces and the theory of best approximations", Russian Math. Surveys, 15:3 (1960), 75-111.

[33] Р. С. Исмагилов, "Поперечники множеств в линейных нормированных пространствах и приближение тригонометрическими многочленами", УМH, 29:3(177) (1974), 161-178; англ. пер.: R. S. Ismagilov, "Diameters of sets in normed linear spaces and the approximation of functions by trigonometric polynomials", Russian Math. Surveys, 29:3 (1974), 169-186.

[34] Б. С. Кашин, "Поперечники некоторых конечномерных множеств и классов гладких функций", Изв. АН СССР. Сер. матем., 41:2 (1977), 334-351; англ. пер.: B.S. Kashin, "Diameters of some finite-dimensional sets and classes of smooth functions", Math. USSR-Izv., 11:2 (1977), 317-333.

[35] Е. Д. Глускин, "Нормы случайных матриц и поперечники конечномерных множеств", Матем. сб., 120(162):2 (1983), 180-189; англ. пер.: E. D. Gluskin, "Norms of random matrices and widths of finite-dimensional sets", Math. USSR-Sb., 48:1 (1984), 173-182.

[36] R. A. DeVore, R. C. Sharpley, S. D. Riemenschneider, " $n$-widths for $C_{p}^{\alpha}$ spaces", $A n$ niversary volume on approximation theory and functional analysis (Oberwolfach, 1983), Internat. Schriftenreihe Numer. Math., 65, Birkhäuser, Basel, 1984, 213-222.

[37] М.Ш. Бирман, М. З. Соломяк, "Кусочно-полиномиальные приближения функций классов $W_{p}^{\alpha}$ ", Матем. сб., 73(115):3 (1967), 331-355; англ. пер.: M. Sh. Birman, M.Z. Solomyak, "Piecewise-polynomial approximations of functions of the classes $W_{p}^{\alpha ", ~ M a t h . ~ U S S R-S b ., ~ 2: 3 ~(1967), ~ 295-317 . ~}$

[38] A. El Kolli, " $n$-ième épaisseur dans les espaces de Sobolev", J. Approximation Theory, 10:3 (1974), 268-294.

[39] И. В. Бойков, “Аппроксимация некоторых классов функций локальными сплайнами”, Ж. вычисл. матем. и матем. физ., 38:1 (1998), 25-33; англ. пер.: I. V. Bojkov, "Approximation of some classes of functions by local splines", Comput. Math. Math. Phys., 38:1 (1998), 21-29.

[40] I. V. Boykov, Optimal approximation and Kolmogorov widths estimates for certain singular classes related to equations of mathematical physics, 2013, arXiv: 1303.0416v1.

[41] H. Triebel, "Entropy and approximation numbers of limiting embeddings; an approach via Hardy inequalities and quadratic forms", J. Approx. Theory, 164:1 (2012), 31-46.

[42] A. A. Vasil'eva, "Widths of weighted Sobolev classes on a John domain: strong singularity at a point", Rev. Mat. Complut., 27:1 (2014), 167-212.

[43] М. С. Айтенова, Л.К. Кусаинова, "Об асимптотике распределения аппроксимативных чисел вложений весовых классов Соболева. I", Матем. журн., 2:1(3) (2002), 3-9 (electronic).

[44] М. С. Айтенова, Л.К. Кусаинова, "Об асимптотике распределения аппроксимативных чисел вложений весовых классов Соболева. II", Матем. журн., 2:2(4) (2002), 7-14 (electronic). 
[45] П.И. Лизоркин, М. О. Отелбаев, “Оценки аппроксимативных чисел оператора вложения для пространств соболевского типа с весами”, Исследования по теории дифферениируемых функиий многих переменных и ее приложениям. Часть 10, Сборник работ, Тр. МИАН СССР, 170, 1984, 213-232; англ. пер.: P. I. Lizorkin, M. O. Otelbaev, "Estimates of approximate numbers of the imbedding operators for spaces of Sobolev type with weights", Proc. Steklov Inst. Math., 170 (1987), 245-266.

[46] М. О. Отелбаев, "Оценки поперечников по Колмогорову для одного класса весовых пространств”, Докл. АН СССР, 235:6 (1977), 1270-1273; англ. пер.: M. Otelbaev, "Estimates of the Kolmogorov diameters for a class of Besov spaces", Soviet Math. Dokl., 18:4 (1978), 1159-1163.

[47] О.В.Бесов, В. П. Ильин, С. М. Никольский, Интегральные представления функиий и теоремы вложения, 2-е изд., Наука, Физматлит, М., 1996, 480 с.; англ. пер. 1-го изд.: O. V. Besov, V. P. Il'in, S. M. Nikol'skii, Integral representations of functions and imbedding theorems, v. I, II, Scripta Series in Mathematics, V.H. Winston \& Sons, Washington, D.C.; Halsted Press [John Wiley \& Sons], New York-Toronto, Ont.-London, 1978, 1979, viii+345 pp., viii+311 pp.

[48] В.Д. Степанов, “Двухвесовые оценки интегралов Римана-Лиувилля", Изв. AH CCCP. Сер. матем., 54:3 (1990), 645-656; англ. пер.: V.D. Stepanov, "Two-weighted estimates of Riemann-Liouville integrals", Math. USSR-Izv., 36:3 (1991), 669-681.

[49] С.Л. Соболев, “Об одной теореме функционального анализа”, Матем. сб., 4(46):3 (1938), 471-497; англ. пер.: S. Sobolev, "On a theorem of functional analysis", Amer. Math. Soc. Transl. (2), 34, 1963, 39-68.

[50] С. Л. Соболев, Некоторые применения функиионального анализа в математической физике, Изд-во ЛГУ, Л., 1950, 255 с.; англ. пер.: S. L. Sobolev, Applications of functional analysis in mathematical physics, Transl. Math. Monogr., 7, Amer. Math. Soc., Providence, R.I., 1963, vii+239 pp.

[51] A. A. Vasil'eva, "Kolmogorov and linear widths of the weighted Besov classes with singularity at the origin", J. Approx. Theory, 167 (2013), 1-41.

[52] А. А. Васильева, "Поперечники весовых классов Соболева на области, удовлетворяющей условию Джона", Ортогональные ряды, теория приближений и смежные вопросы, Сборник статей. К 60-летию со дня рождения академика Бориса Сергеевича Кашина, Тр. МИАН, 280, МАИК, М., 2013, 97-125; англ. пер.: A. A. Vasil'eva, "Widths of weighted Sobolev classes on a John domain", Proc. Steklov Inst. Math., 280 (2013), 91-119.

\section{Анастасия Андреевна Васильева (Anastasia A. Vasil'eva)}

Поступила в редакцию

Математический институт им. В.А. Стеклова

29.12.2014 и 26.06.2015

Российской академии наук, г. Москва

E-mail: vasilyeva_nastya@inbox.ru 